\title{
Phonon-assisted radiofrequency absorption by gold nanoparticles resulting in hyperthermia
}

\author{
Andrei Postnikov \\ Université de Lorraine, \\ LCP-A2MC, 1 Bd Arago, \\ F-57078 Metz, \\ France* \\ Kamil Moldosanov \\ Kyrgyz-Russian Slavic University, \\ 44 Kiyevskaya St., \\ Bishkek 720000, \\ Kyrgyzstar
}

It is suggested that in gold nanoparticles (GNPs) of about $5 \mathrm{~nm}$ sizes used in the radiofrequency $(\mathrm{RF})$ hyperthermia, an absorption of the RF photon by the Fermi electron occurs with involvement of the longitudinal acoustic vibrational mode (LAVM), the dominating one in the distribution of vibrational density of states (VDOS). This physical mechanism helps to explain two observed phenomena: the size dependence of the heating rate (HR) in GNPs and reduced heat production in aggregated GNPs. The argumentation proceeds within the one-electron approximation, taking into account the discretenesses of energies and momenta of both electrons and LAVMs. The heating of GNPs is thought to consist of two consecutive processes: first, the Fermi electron absorbs simultaneously the RF photon and the LAVM available in the GNP; hereafter the excited electron gets relaxed within the GNP's boundary, exciting a LAVM with the energy higher than that of the previously absorbed LAVM. GNPs containing the Ta and/or Fe impurities are proposed for the RF hyperthermia as promising heaters with enhanced HRs, and GNPs with rare-earth impurity atoms are also brought into consideration. It is shown why the maximum $\mathrm{HR}$ values should be expected in GNPs with about $5-7 \mathrm{~nm}$ size.

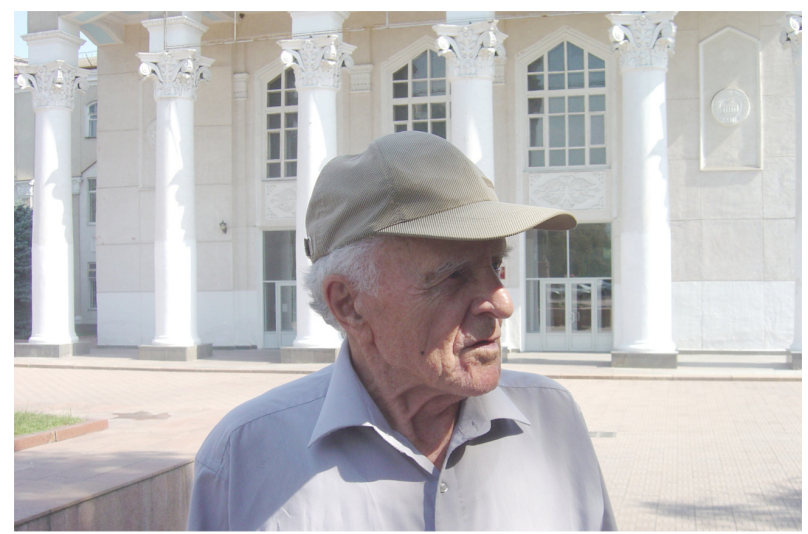

The authors dedicate this work to the 90th anniversary of professor Leonid V. Tuzov of the Kyrgyz State University

* andrei.postnikov@univ-lorraine.fr

$\dagger$ altair1964@yandex.ru 


\section{INTRODUCTION}

Among methods allowing for hyperthermia in biological tissues, that based on the radiofrequency (RF) absorption by gold nanoparticles (GNPs) got in the last years much attention, due to its important advantage - a deep penetration of the RF radiation into biological tissue $(\sim 30 \mathrm{~cm}$ at frequencies $\sim 10 \mathrm{MHz})$. Details and useful references can be found in the review by Corr et al. (2012b). Moran et al. $(2009)$ in their experiments carried out at $13.56 \mathrm{MHz}$ revealed that the heating rate (HR) depends on the particle size, namely that smaller particles are heated faster, presumably because of their higher resistivity. However, the exact physical basis of heat generation by nanoparticles remained so far unclarified. This is a serious obstacle for grasping the HR / particle size relation and hence for making "intelligent choice" of the GNP size, tailored to the particular use. For example, one may wish to assure an enhanced specific absorption rate (SAR) to heat the GNP to necessary temperatures at minimum released power, avoiding at the same time an unnecessary heating of normal tissues during hyperthermia.

According to standard definition, $\operatorname{SAR}=C(\Delta T / \Delta t)$, where $C$ is the specific heat and $\Delta T / \Delta t$ is the HR (temperature / time). The size dependence of the HR for the GNPs used by Moran et al. (2009) is presented in Fig. 1. As is seen, the HR attains maximal values, among the nanoparticles studied, in the smallest ones, of the $5 \mathrm{~nm}$ size. Along with Moran et al. (2009), also the observations of Kruse et al. (2011); Raoof and Curley (2011) imply that the smaller the size of GNPs (for a given volume fraction of gold), the higher their HR: among experimentally studied GNPs with diverse sizes, those of $\sim 5 \mathrm{~nm}$ diameter exhibit the highest one. Corr et al. (2012a) strengthened this conclusion, having stated that the GNPs only of size $10 \mathrm{~nm}$ or less could be heated by the electromagnetic RF field (again, the highest experimentally observed HR corresponded to the GNPs of $5 \mathrm{~nm}$ diameter).

In this paper, a physical model of the size effect in heat generation and reduction of heat generation in aggregated GNPs is proposed, in which the longitudinal acoustic vibrational modes (LAVMs) play an important role. None of the theoretical models reviewed critically by Corr et al. (2012b) took these latter into account, whereas we will show that the inclusion of acoustic modes enables to explain a number of experimental results. Besides, our findings make it possible to obtain an optimal size of GNPs providing maximum SAR.

The manuscript is organised as follows. Sec. II shows that in large GNPs (of the diameter exceeding $162 \mathrm{~nm}$ ) the heating by RF radiation is possible owing to uncertainty in the Fermi electron's momentum; however, the use of this mechanism in hyperthermia cannot be but very limited. For practical treatment, much smaller GNPs need to be - and indeed are - used, revealing a pronounced size dependence of the heating efficiency. Possible physical foundations of the related heating mechanism, along with explanation

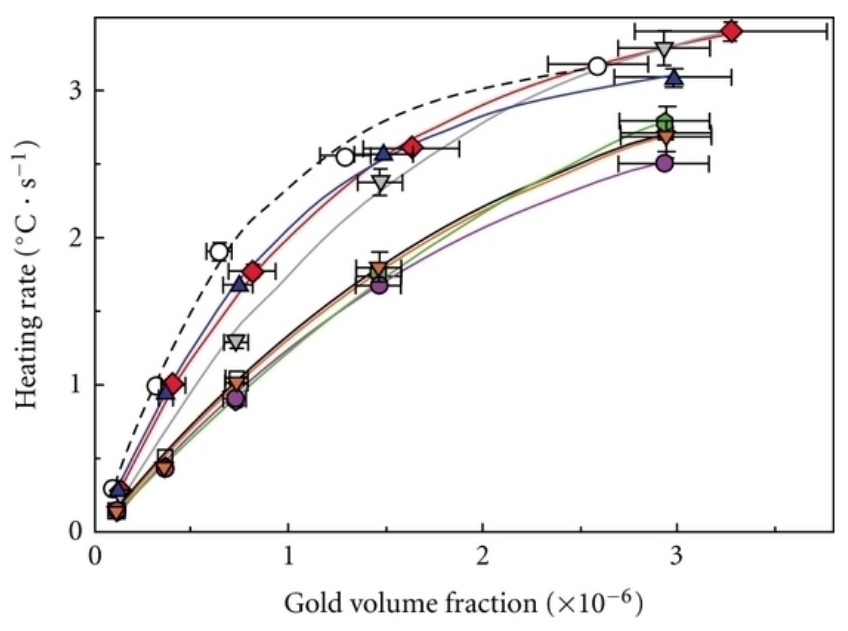

$\begin{array}{ll}\bigcirc 150 \mathrm{~nm} \text { nanoshell } & \square 50 \mathrm{~nm} \text { gold } \\ \diamond 5 \mathrm{~nm} \text { gold } & \bigcirc 100 \mathrm{~nm} \text { gold } \\ \Delta 10 \mathrm{~nm} \text { gold } & \bigcirc 150 \mathrm{~nm} \text { gold } \\ \nabla 20 \mathrm{~nm} \text { gold } & \nabla 250 \mathrm{~nm} \text { gold }\end{array}$

FIG. 1 Size-dependent heating of gold nanoparticles (diameters indicated) in the RF field of the frequency 13.56 MHz. Reproduced with permission from Moran et al. (2009). 
of the observed size effect, are discussed in Sec. III. In Sec. IV on the basis of conclusions drawn from Sec. III. the experimentally observed reduced heat production in aggregated GNPs is explained. Sec. V addresses possible ways of further increase of the HP in GNPs. The general discussion of results is offered in Sec. VI, and the conclusion drawn in Sec. VII.

\section{SETTING THE STAGE; CONDITIONS FOR THE RF ABSORPTION}

First we discuss an issue of whether the phonons are at all needed for the RF absorption, and what are the conditions for the latter to happen in a "conventional" way. The key feature in absorption of electromagnetic waves by nanoparticles is quantization of energy levels, which become denser as the particle size increases. Satisfying the conditions of conservation of both energy and momentum on absorption is not, a priori, possible without additional considerations, because the curvature/slope of dispersion relations are very different for electrons and photons. We consider first the case when no other players enter the picture, and assume the matching conditions to be satisfied within the uncertainty relation. To be specific, we take the GNP to be spherical, of diameter $D$; the $\mathrm{RF}$ of $13.56 \mathrm{MHz}$, like in the above experiment, corresponds to photon energy $h \nu=5.6 \cdot 10^{-8} \mathrm{eV}$ and momentum $p_{\mathrm{ph}} \approx 3 \cdot 10^{-30} \mathrm{~g} \cdot \mathrm{cm} \cdot \mathrm{s}^{-1}$.

The skin depth in gold at $13.56 \mathrm{MHz}$ is about $20 \mu \mathrm{m}$, i.e., by orders of magnitude exceeding the GNP size. One can therefore assume that the electric field penetrates the volume and is of the same strength throughout the particle.

In metal particles as small as $\sim 5 \mathrm{~nm}$, and even for much larger ones, the quantization (discreteness) of the electron energy spectrum is a crucial factor shaping their properties. Speaking specifically about gold, one can advance far enough on the basis of the free-electron model, assuming one electron per atom and estimating the corresponding Fermi energy.

Assume that the absorption happens due to transitions between discrete levels induced by quantum confinement; the free-electron dispersion relation $E=p^{2} / 2 m$ implies that the energy step $\Delta E=E_{\mathrm{ph}}$ must be accompanied by the correction of momentum, $\Delta p_{\mathrm{F}} \approx E_{\mathrm{ph}} m / p_{\mathrm{F}}=h \nu / v_{\mathrm{F}}$ (see Fig. 2). For the relevant values of $h \nu=5.6 \cdot 10^{-8} \mathrm{eV}$ and the Fermi velocity of gold as in the free-electron model $\left(v_{\mathrm{F}} \approx 1.4 \cdot 10^{6} \mathrm{~m} \cdot \mathrm{s}^{-1}\right)$, the momentum mismatch amounts to $\Delta p_{\mathrm{F}} \approx 6 \cdot 4 \cdot 10^{-28} \mathrm{~g} \cdot \mathrm{cm} \cdot \mathrm{s}^{-1}$, two orders of magnitude larger than what the photon momentum (see above) could bring about. However, the small size of GNP may result in appreciable Heisenberg's uncertainty of electron momenta in them and thus "smear out" the discussed mismatch condition. Let us make some estimates to this end.

Following the pioneering works by (Kubo, 1962, 1977), we remind a relationship between the diameter $D$ and the level spacing $\Delta E$. Assume that the electron energy level $E^{\prime}$ is the first vacant one above the Fermi level $E_{\mathrm{F}}$. The level spacing $\Delta E_{\mathrm{el}}=E^{\prime}-E_{\mathrm{F}}$ and the number of atoms $N_{a}$ in the GNP are related as follows (Kubo, 1962, the derivation of the Kubo's formula is given in Appendix A):

$$
\begin{gathered}
\Delta E_{\mathrm{el}} \approx(4 / 3) \cdot E_{\mathrm{F}} / N_{a}, \quad \text { or } \quad N_{a} \approx(4 / 3) \cdot E_{\mathrm{F}} / \Delta E_{\mathrm{el}} \\
D \approx\left(\frac{8}{\pi} \frac{m_{a}}{\rho} \frac{E_{\mathrm{F}}}{\Delta E_{\mathrm{el}}}\right)^{1 / 3},
\end{gathered}
$$

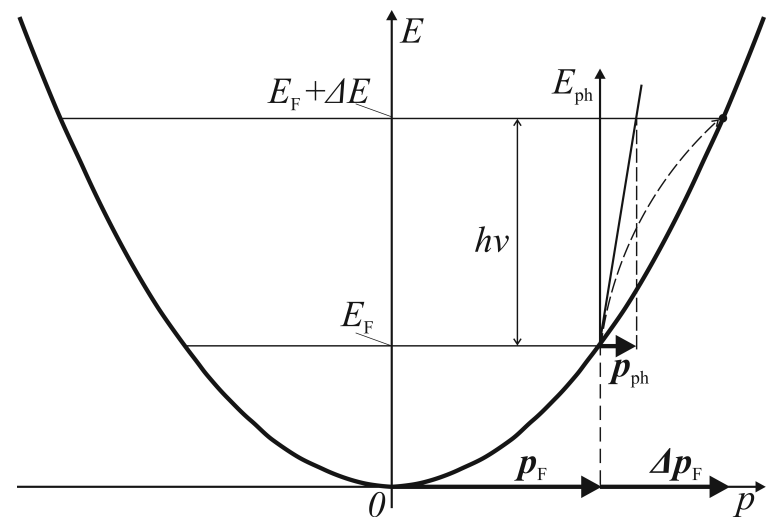

FIG. 2 Scheme of absorption of RF photon by the Fermi electron without involvement of LAVM. Dashed curve indicates a transition of the Fermi electron excited by the RF photon with energy $h \nu$. 


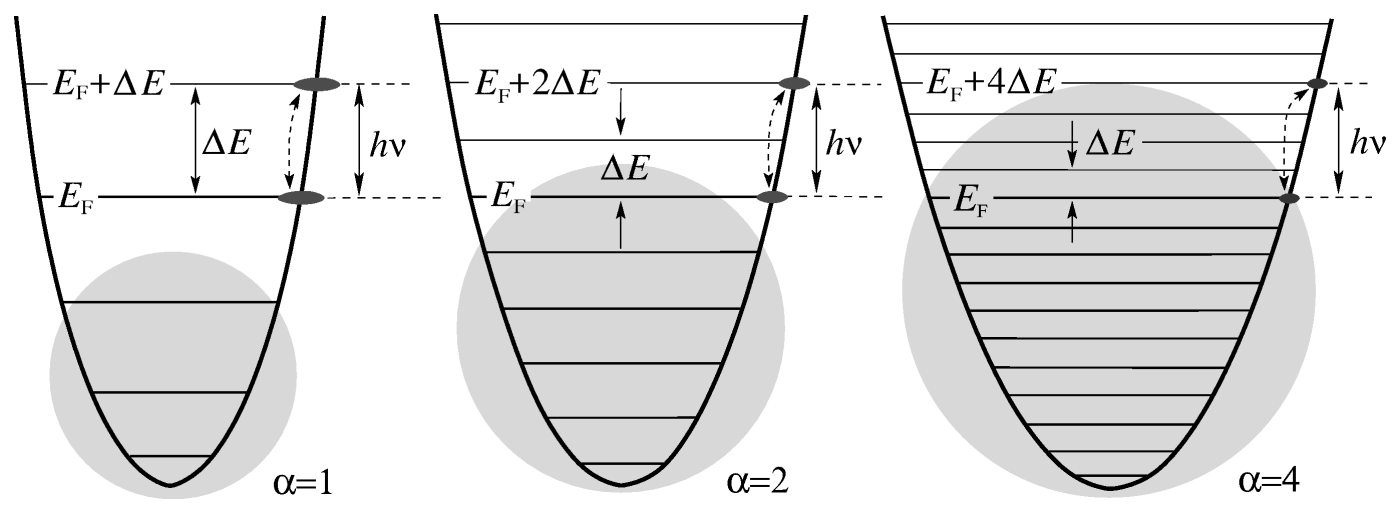

FIG. 3 Schemes of "direct" absorption of RF photons $h \nu$ by electrons at the Fermi level $E_{\mathrm{F}}$, for different energy discretisation steps $\Delta E$, depending on the particle size, and for different step multiplicities $\alpha$ (see text and Table I for details). The uncertainty of the electron's momentum is hinted by elliptical dots of different width.

where $m_{a}$ is the atomic mass of gold, $m_{a}=197$ in atomic units, or $\approx 3.27 \cdot 10^{-22} \mathrm{~g} ; \rho=19.3 \mathrm{~g} \cdot \mathrm{cm}^{-3}$ is the density of gold, $E_{\mathrm{F}}=5.52 \mathrm{eV}$. For $\Delta E_{\mathrm{el}}=h \nu=5.6 \cdot 10^{-5} \mathrm{meV}, D=162.1 \mathrm{~nm}$. Remind that these are estimates for the smallest GNP size which allows the RF absorption at $13.56 \mathrm{MHz}$, from the energy level spacing arguments. Now, we turn to the momentum uncertainty arguments and assume for simplicity that the direction along the nanoparticle's diameter is collinear with the momentum $\Delta p_{\mathrm{F}}$ and the Fermi electron's momentum $\vec{p}_{\mathrm{F}}$. Then the uncertainty in the Fermi electron's momentum in this direction equals $\Delta p \sim h /(2 \pi \cdot D)$. At $D=162.1 \mathrm{~nm}, \Delta p \sim 6.5 \cdot 10^{-23} \mathrm{~g} \cdot \mathrm{cm} \cdot \mathrm{s}^{-1}$, that largely exceeds $\Delta p_{\mathrm{F}} \approx 6.4 \cdot 10^{-28} \mathrm{~g} \cdot \mathrm{cm} \cdot \mathrm{s}^{-1}$. Consequently, the momentum conservation law can be helped by such uncertainty, and the RF photon absorbed. We keep in mind, however, that the minimal GNP size for the direct $\mathrm{RF}$ absorption must be at least $\approx 162.1 \mathrm{~nm}$. Obviously as the particle size grows, the distance between quantified levels shrinks, so that an absorption of a given photon $h \nu$ may bring about an excitation across $\alpha$ (an integer number) interlevel distances. This situation is schematically shown in Fig. 3, which implies a parabolic dispersion law $E=p^{2} / 2 m$ for different confinements (particle sizes), in arbitrary scale. The effect of the momentum uncertainty is indicated by dots of different widths placed at the initial and final levels. Some numerical estimates for the energy spacings and momentum uncertainties, relevant for the absorption of quanta at $\nu=13.56 \mathrm{MHz}\left(h \nu=5 \cdot 6 \cdot 10^{-5} \mathrm{meV}\right)$ are summarized in Table I An issue of matrix elements for such transitions, some of which might be suppressed in the dipole approximation due to symmetry considerations, is left aside. The numerical relation between the energy split multiplicity $\alpha$, given by $\Delta E_{\mathrm{el}}=h \nu / \alpha$, and the diameter of particle (assuming the latter spherical) from Eq. (1) is as follows:

$$
D \approx \alpha^{1 / 3} \cdot\left[(8 / \pi) \cdot\left(m_{a} / \rho\right) \cdot\left(E_{\mathrm{F}} / h \nu\right)\right]^{1 / 3}=\alpha^{1 / 3} \cdot 162.1[\mathrm{~nm}]
$$

TABLE I Parameters of GNPs with level spacings $h \nu / \alpha(\alpha=1,2,4,10$ taken as examples $)$ able to absorb the $13.56 \mathrm{MHz}$ photons thanks to the uncertainties in the Fermi electrons' momenta

\begin{tabular}{llll}
\hline $\begin{array}{l}\text { Number of } \\
\text { level spacings } \alpha\end{array}$ & $\begin{array}{l}\text { Level spacing } \\
h \nu / \alpha\left(10^{-5} \mathrm{meV}\right)\end{array}$ & $\begin{array}{l}\text { Diameter } \\
D_{\alpha}(\mathrm{nm})\end{array}$ & $\begin{array}{l}\text { Uncertainty in Fermi } \\
\text { electron's momentum } \Delta p_{\alpha} \\
\left(10^{-23} \mathrm{~g} \cdot \mathrm{cm} \cdot \mathrm{s}^{-1}\right)\end{array}$ \\
\hline 1 & 5.6 & 162.1 & 6.5 \\
2 & 2.8 & 204.2 & 5.2 \\
4 & 1.4 & 257.3 & 4.1 \\
10 & 0.6 & 349.2 & 3.0 \\
\hline
\end{tabular}


The last column of Table I specifies the corresponding momentum uncertainty, $h /(2 \pi \cdot D)$. In all the cases considered (until entering much larger particle sizes than those covered by Table I), the uncertainty in the Fermi electron's momentum $\Delta p_{\alpha}$ exceeds by far the "adjustment" $\Delta p_{\mathrm{F}} \approx 6.4 \cdot 10^{-28} \mathrm{~g} \cdot \mathrm{cm} \cdot \mathrm{s}^{-1}$, discussed above as a reference value to absorb the momentum mismatch required to promote a Fermi electron onto the energy level $\left(E_{\mathrm{F}}+h \nu\right)$. Thus, the momentum conservation law can be fulfilled, and the $13.56 \mathrm{MHz}$ photons absorbed, for GNPs larger than $\sim 162 \mathrm{~nm}$. Consequently the latter, as it seems, can apparently be RF-heated "directly", i.e., without involvement of LAVMs, just by exciting the Fermi electrons to the available energy levels above the Fermi level. On the contrary, in GNPs smaller than $162 \mathrm{~nm}$ the separation between adjacent levels exceeds the energy of the RF quantum, and at "working" particle sizes which in fact show an enhanced absorption $(\sim 5 \mathrm{~nm})$ the level splitting becomes prohibitively large. An involvement of many photons in a single electron excitation is likely to be a very rare process. Therefore, we have to bring another physical mechanism into consideration. Longitudinal acoustic phonons seem to be able to intervene with energies of the "correct" order of magnitude. The prerequisites of their practical involvement are discussed in the following.

\section{LAVM-ASSISTED ABSORPTION OF RF PHOTON BY A FERMI ELECTRON}

The observed size dependence of the HR can be explained by a mechanism of the GNPs' heating that attributes a crucial role in the absorption of a RF photon to LAVM. To make reliable estimates, we need to know something about elastic properties of nanoparticles. A theoretical work by D.Y. Sun et al. (2001) demonstrated that the metallic nanoparticles retain the bulklike core region. Experimentally, J. Sun et al. (2014) have recently found that Ag nanoparticles can be deformed like liquid droplets but remain highly crystalline in the interior.

As was already admitted above, we assume the GNP to be spherical; we remind that the RF of $13.56 \mathrm{MHz}$ corresponds to photon energy $h \nu=5 \cdot 6 \cdot 10^{-8} \mathrm{eV}$ and momentum $p_{\mathrm{ph}} \approx 3 \cdot 10^{-30} \mathrm{~g} \cdot \mathrm{cm} \cdot \mathrm{s}^{-1}$. The absorption happens due to transitions between discrete levels induced by quantum confinement, as was elaborated above and schematically shown in Fig. 3 .

From now on, we turn to discussing a case of nanoparticles too small for a RF phonon energy to "bridge the gap" between largely split quantified levels. We bring into consideration a scheme of absorption of a RF photon whereby a Fermi-level electron excitation is helped by an involvement of a LAVM. A "classical" view onto the electron-phonon interaction (otherwise straightforwardly grasped in terms of energy and momentum exchange between the corresponding quasiparticles) is that the electrons are driven by, or themselves contribute to, the fluctuating electric field due to compression / dilation of the electron density in the course of lattice vibrations. Obviously, only the longitudinal vibration mode can be "useful" in this sense. Its wavevector moreover must be reasonably far from the Brillouin zone (BZ) center, where the dispersion starts to bend "flat" and to yield high density of modes 1 1

A Fermi electron may absorb the energies of both the RF photon and the LAVM. Fig. 4 shows how an inclusion of momentum $n_{\mathrm{vm}} \cdot q$ and energy $n_{\mathrm{vm}} \Delta E_{\mathrm{vm}}$ of such LAVM $\left(n_{\mathrm{vm}}\right.$ numbers the energy steps in the vibration modes) into the combined absorption makes possible to satisfy both conservation laws. In the absorption event, the GNP borrows energy from its LAVMs system, adds it to that of the RF quantum and excites an electron to the vacant level beyond the $E_{\mathrm{F}}$ :

$$
E_{\mathrm{F}}+\Delta E_{\mathrm{vm}}+h \nu=\left(\vec{p}_{\mathrm{F}}+\vec{q}+\vec{p}_{\mathrm{ph}}\right)^{2} / 2 m
$$

where $E_{\mathrm{F}} / \vec{p}_{\mathrm{F}}, \Delta E_{\mathrm{vm}} / \vec{q}$ and $h \nu / \vec{p}_{\mathrm{ph}}$ are the energy / momentum of the free electron, the LAVM and photon, correspondingly (see Fig. 4p, and $m$ is the electron's mass. We note, for the sake of a later reference, that an electron may well be excited across $m_{\mathrm{el}}>1$ steps of its discrete spectrum, i.e., onto $E_{\mathrm{F}}+m_{\mathrm{el}} \Delta E_{\mathrm{el}}$, that is however not explicitly depicted in Fig. 4. Once excited, the propagating mobile electron very likely will be trapped (relaxed) before reaching the GNP surface (see Subsec. III.C on the mean free path issues), releasing more energy to the LAVMs pool than was borrowed beforehand. The net effect of that is the heating of the GNP.

\footnotetext{
${ }^{1}$ A numerical estimate can be drawn from (Singh and Ali, 2013), who studied the phonon dispersions in amorphous metals. For gold, the first maxiumum of the $\omega(q)$ dispersion occurs at $q \sim 1.5 \AA^{-1}$ whereas up to $q \sim 1.0 \AA^{-1}$ the dispersion remains reasonably linear.
} 


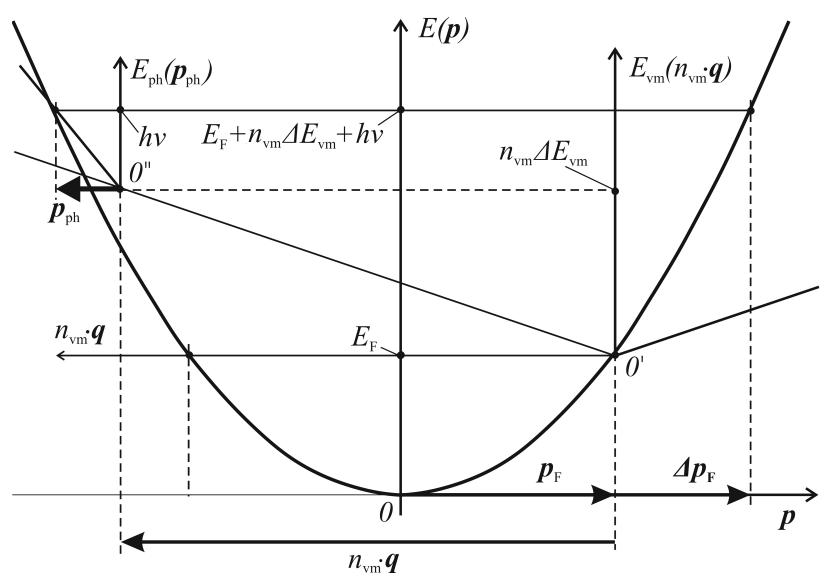

FIG. 4 Scheme of absorption of RF photon by the Fermi electron assisted by an absorption of a LAVM (in arbitrary scale). The dispersion laws for photons $E_{\mathrm{ph}} \sim p_{\mathrm{ph}}$, electrons $E(p) \sim p^{2} / 2 m$ and LAVMs $E_{\mathrm{vm}} \sim\left(n_{\mathrm{vm}} \cdot q\right)$ are shown. See text for details.

In Fig. 4 a point on the Fermi sphere (identified by momentum $\vec{p}_{\mathrm{F}}$ and energy $E_{\mathrm{F}}$ ) serves as an origin of the LAVM dispersion branch, on which the momentum $n_{\mathrm{vm}} \cdot \vec{q}$ and the mode energy $\Delta E_{\mathrm{vm}}$ stand for some "representative" mode. Let us give some numerical estimates to these parameters.

The free-electron model for bulk gold yields the Fermi radius $k_{\mathrm{F}}=1.20 \cdot 10^{10} \mathrm{~m}^{-1}$, quite close to the mean experimental estimates for its slightly non-spherical belly (Coleridge and Templeton, 1982). The corresponding free-electron Fermi energy is $E_{\mathrm{F}}=5.52 \mathrm{eV}$; the Kubo formula for the level splitting at the Fermi energy due to spatial confinement $\Delta E_{\mathrm{vm}}$ yields, for the diameter $D=5 \mathrm{~nm}, \Delta E_{\mathrm{vm}}=1.91 \mathrm{meV}$, and the corresponding increment of the electron's momentum to bring it to the first vacant level is $\Delta k_{\mathrm{F}}=$ $2.08 \cdot 10^{6} \mathrm{~m}^{-1} \approx 0.017$ of the $k_{\mathrm{F}}$. As RF $h \nu=5 \cdot 6 \cdot 10^{-5} \mathrm{meV} \ll \Delta E_{\mathrm{vm}}$, the larger part of the latter gap has to be overcome, according to out hypothesis, by borrowing energy from LAVM. From the velocity of sound in gold (3240 $\mathrm{m} \cdot \mathrm{s}^{-1}$ longitudinal, $1200 \mathrm{~m} \cdot \mathrm{s}^{-1}$ transversal), the highest phonon energy extrapolated onto the nearest BZ boundary, i.e., the $L$ point, is $\sim 28.5 \mathrm{meV}\left(L_{\mathrm{L}}\right)$ and $\sim 10.5 \mathrm{meV}\left(L_{\mathrm{T}}\right)$. Corresponding experimental frequencies at $L$ are bended downwards as expected, to, correspondingly, 19.1 and $7.7 \mathrm{meV}$ (as cited by Dal Corso, 2013, see also his first-principles calculation of vibration spectrum). Consequently, the energy matching to $\Delta E_{\mathrm{vm}}$ can be realized via an interaction with an acoustic phonon whose momentum is within $\sim 7-18 \%$ of the BZ radius. Inversely, an interaction with a phonon close to the BZ periphery may, in principle, promote an electron onto a higher (up to the 3th or 4th) energy state beyond the Fermi level. Two observations, however, need to be stressed in this relation. First, the numerical relations in gold (see above) are such that matching the energies of electron and phonon is impossible if their momenta stay collinear, as Fig. 4 implies. A more realistic scenario, discussed below, comprises $\vec{k}_{\mathrm{F}}$ staying at some angle to the phonon momentum $\vec{q}$. The second observation imposes the quantisation of phonon $\vec{q}$ values in a nanoparticle as $q=h / L$, where $L$ is a length of the chain of ions along which the LAVM propagates. As an estimate of the order of magnitude, the relevant length is between the nanopaticle's diameter $(5 \mathrm{~nm})$ and circumference, hence the $q$ step is $\Delta q=[1 \cdots 2 \pi] / L=[0.2 \cdots 1.26] \cdot 10^{9} \mathrm{~m}^{-1}$, i.e., $\sim[2 \cdot 11] \%$ of $k_{\text {F }}$.

In the following, we'll often refer to circular contour / path over which the compression mode propagates, as this is a simple yet realistic model case. Although the natural diversity of nanoparticle sizes and shapes makes a faithful simulation difficult, the circular path has a virtue of being the longest one in a "round" particle, hence hosting the maximal number of modes, densely distributed in the $\vec{q}$ space. Consequently, the quantified energies of modes on a circular contour make a denser spectrum than those on any other path; within a given energy interval, more individual modes can be found and used for borrowing energy to an electron. It will be argued below that longitudinal acoustic phonon cannot propagate strictly on the surface, but rather at a (small) depth.

We discuss now the impact of the electron and phonon momenta being non-collinear, but, in any case, coplanar. A more realistic scheme in this sense than that of Fig. 4 is shown in Fig. 5 , where however the energy and the momentum of the RF photon are neglected, by force of relations $h \nu \ll E_{\mathrm{vm}} ; p_{\mathrm{ph}} \ll n_{\mathrm{vm}} q$; $p_{\mathrm{ph}} \ll p_{\mathrm{F}}$. Fig. 5 depicts therefore a possibility of absorption, by electrons at the Fermi surface, of the LAVMs with energies $\Delta E_{\mathrm{vm}}, 2 \Delta E_{\mathrm{vm}}, 3 \Delta E_{\mathrm{vm}}$, and corresponding momenta, within the same nanoparticle of a given size. It is (arbitrarily, just for the sake of simplifying the figure) implied that $m_{\mathrm{el}}=n_{\mathrm{vm}}$ and hence $\Delta E_{\mathrm{el}}=\Delta E_{\mathrm{vm}}$, therefore the augmentation of the electron energy on absorption happens in portions of $\Delta E_{\mathrm{vm}}$. 


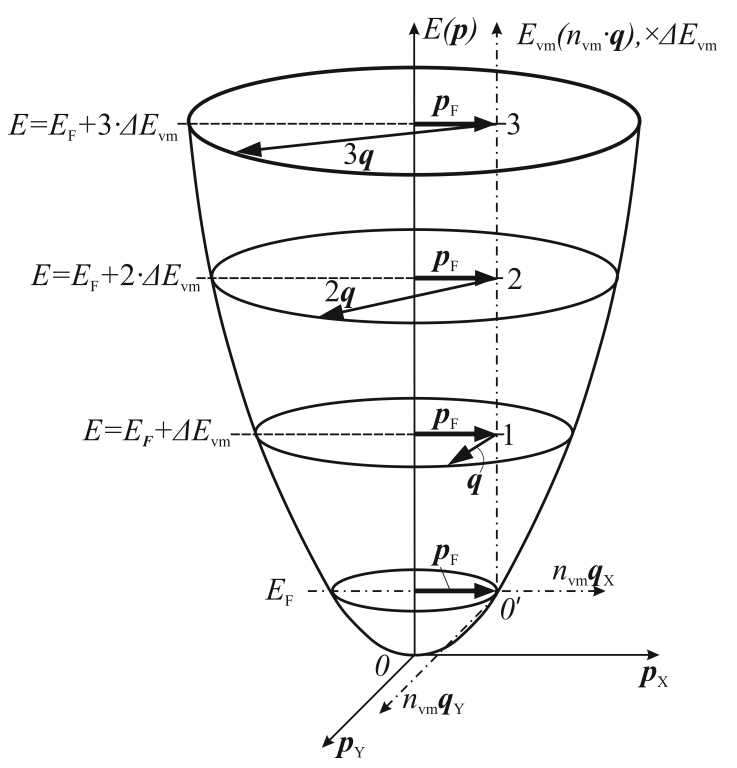

FIG. 5 Same as in Fig. 4. however assuming $m_{\mathrm{el}}=n_{\mathrm{vm}}$ and $\Delta E_{\mathrm{el}}=\Delta E_{\mathrm{vm}}$, for the general case of the electron momentum $\vec{p}_{\mathrm{F}}$ and the phonon momentum $\vec{q}$ being coplanar. For simplicity, the energy and the momentum of the RF photon are neglected. See text for details.

A more detailed projection of the momenta matching, involving also the RF photon momentum, is shown in Fig. 6. Possible excitations from an initial state $\vec{p}_{\mathrm{F}}$ of energy $E_{\mathrm{F}}$ via an absorption of a phonon $\vec{q}$ and a RF photon $\vec{p}_{\mathrm{ph}}$ end up in a state with the energy $E=E_{\mathrm{F}}+\Delta E_{\mathrm{vm}}+h \nu$. The allowed "chained" $\vec{p}_{\mathrm{F}} \cdots \vec{q} \cdots \vec{p}_{\mathrm{ph}}$ vectors fall within a body of revolution around the fixed $\vec{p}_{\mathrm{ph}} \| \vec{p}_{x}$ direction, limited on the left by the cone of [side $\vec{p}_{\mathrm{F}} /$ aperture $2 \beta$ ] and on the right - by the spherical (radius $\left|\vec{p}_{\mathrm{F}}+\vec{q}+\vec{p}_{\mathrm{ph}}\right|$ ) cape built on top of the cylinder of the height $p_{\mathrm{ph}}$. The conical and cylindrical surface parts are connected by the intermediate conical belt of the width $q$. From Fig. 6(a), the maximal angle $\beta$ the $\vec{p}_{\mathrm{F}}$ vector may build to $\vec{q}$ is

$$
\beta=\arccos \frac{\left(\sqrt{2 m_{e} E}-q\right)^{2}-p_{\mathrm{ph}}^{2}-p_{\mathrm{F}}^{2}}{2\left(\vec{p}_{\mathrm{ph}} \cdot \vec{p}_{\mathrm{F}}\right)} .
$$

On relaxation of the excited electron back to $E_{\mathrm{F}}$, the extra energy $E^{\prime}=\Delta E_{\mathrm{vm}}+h \nu$ is released into the

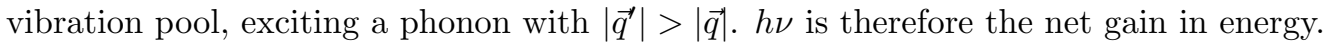
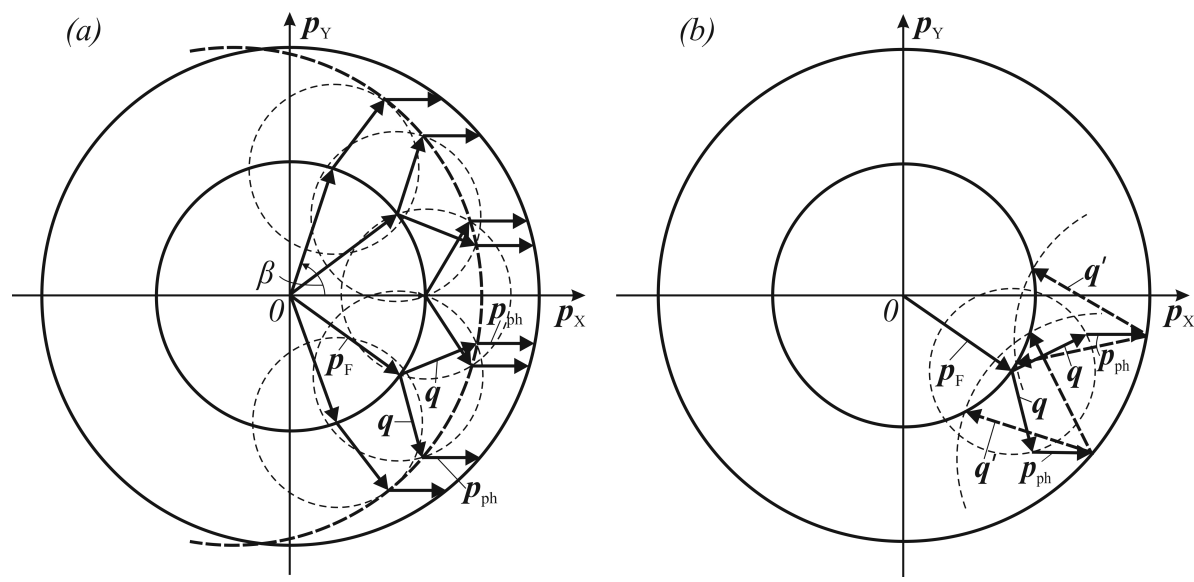

FIG. 6 Projection of the vector construction from Fig. 5 onto the plane comprising the $\vec{p}_{\mathrm{F}}$ and $\vec{q}$ vectors; the photon momentum $\vec{p}_{\mathrm{ph}}$, neglected in Fig. 5 , is now retained. Circles centered at the origin of the coordinate system, of radii $\left|\vec{p}_{\mathrm{F}}\right|$ and $\left|\vec{p}_{\mathrm{F}}+\vec{q}+\vec{p}_{\mathrm{ph}}\right|$, are cross-sections of the electron energy paraboloid at $E=E_{\mathrm{F}}$ and $E=E_{\mathrm{F}}+\Delta E_{\mathrm{vm}}+h \nu$. (a): Some examples of matching momenta and energies in the process of electron excitation from $\vec{p}_{\mathrm{F}}$ due to absorption of phonon $\vec{q}$ and RF photon $\vec{p}_{\mathrm{ph}}$. $(b)$ : For one of $\vec{p}_{\mathrm{F}}$ vectors from panel $(a)$, excitation channels are followed by possible relaxation channels into the initial state via release of phonon $\vec{q}^{\prime}$. Note that $\left|\vec{q}^{\prime}\right|>|\vec{q}|$. 


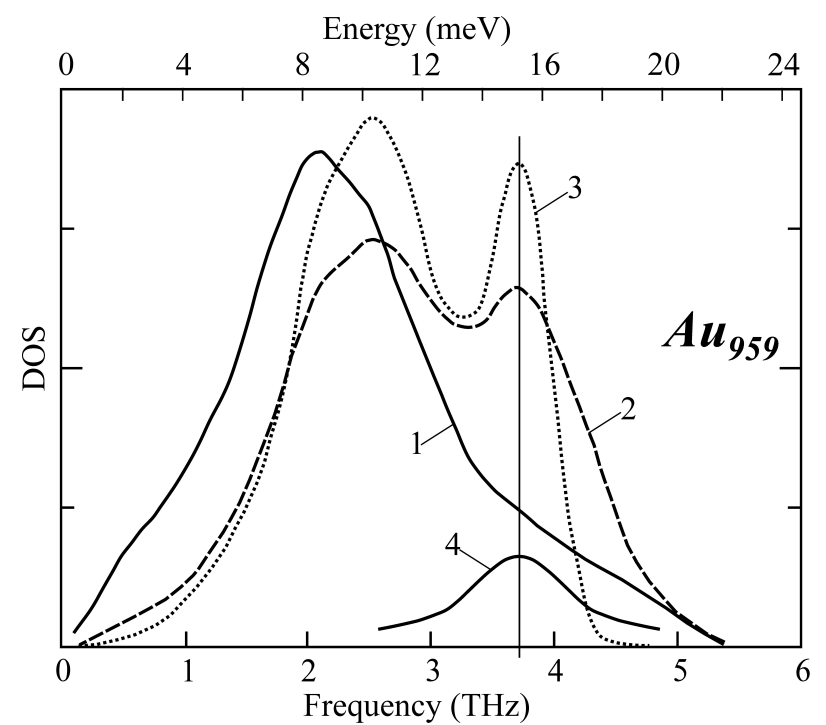

FIG. 7 VDOS in Aug59 nanocrystal, reproduced with our modification with permission from (Sun et al. 2001). Curve 1: contribution from the surface shell; curve 2: from the transition shell, curve 3: from the core region; curve 4: our approximate extraction of the VDOS for LAVMs in the core region, yielding a peak centered at $3.7 \mathrm{THz}(15.3 \mathrm{meV})$, with full width at half maximum $(\mathrm{FWHM}) \sim 1 \mathrm{THz}(\sim 4.14 \mathrm{meV})$.

In the following, we consider the LAVM within a GNP as a compression wave propagating along a closed chain of atoms, in the spirit of the Born - von Kármán cyclic boundary conditions. For this analysis, the work by D.Y. (Sun et al. , 2001) is useful which deals with elastic properties and vibrational density of states (VDOS) in GNPs, indicating notably three structure elements of a nanoparticle: the surface shell (of $\approx 2 \AA$ thickness), the transition shell (of $\approx 3 \AA$ thickness beneath) and the core region. Fig. 7 depicts the corresponding decomposition of VDOS for the case of 959-atom GNP, of the diameter $\approx 3.2 \mathrm{~nm}$. In the core and transition shell, the distribution of VDOS clearly shows the peaks at $\approx 3.7 \mathrm{THz}\left(E_{\mathrm{vm}} \approx 15.3 \mathrm{meV}\right)$, resembling the peak in the VDOS of bulk gold in the experimental work by (Muñoz et al. 2013), which we attribute as being due to LAVMs (see Fig. 8). The VDOS of the surface shell in Fig. 7 does also indicate a feature at the corresponding energy, that we extracted from the background as the curve 4 in Fig. 7 .

The VDOS maximum under discussion is strongest in the core region, which is therefore expected to contribute mostly to the RF absorption. According to D.Y. (Sun et al., 2001), the core region is bulklike, therefore while discussing its contribution to the RF absorption, one can rely on the density and sound velocity values for crystalline gold. This is consistent with experimental observations on the gold's homologue, silver, by J. (Sun et al. 2014) who have found that its nanoparticles remain highly crystalline in the interior. In the following discussion, we assume that the "useful" LAVMs propagate at the depth $\delta=0.7 \mathrm{~nm}$, i.e., along the closed contour entirely within the bulklike core region.

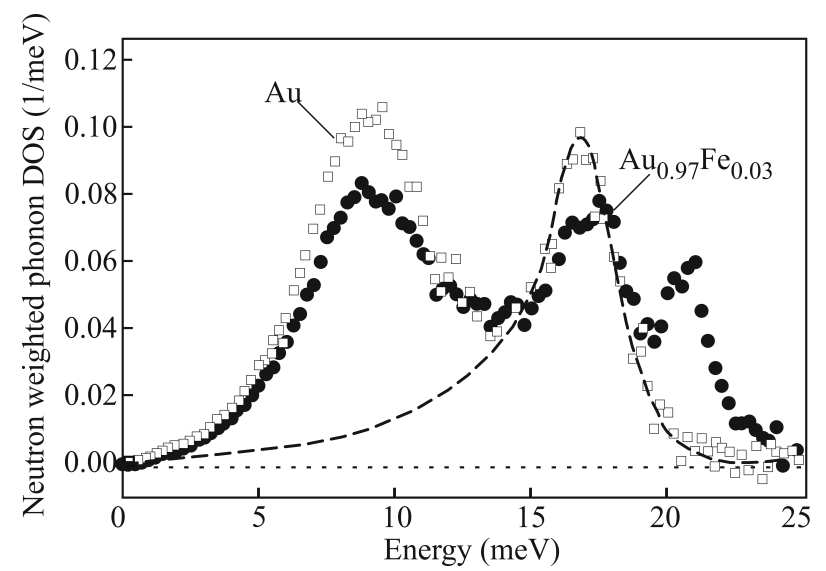

FIG. 8 Experimental phonon DOS curves for bulk pure $\mathrm{Au}$ and $\mathrm{Au}_{0.97} \mathrm{Fe}_{0.03}$ from the inelastic neutron scattering measurements; reproduced with permission from (Muñoz et al. 2013). Dashed line is our approximate extraction of the VDOS curve for LAVMs (the FWHM range is from 14.6 to $18.4 \mathrm{meV}$; the peak position is at $16.8 \mathrm{meV})$. 


\section{A. Energy conservation}

We discuss now some quantization relations for electrons and LAVMs following from the GNP geometry. The energy conservation condition for the absorption of a RF photon with the energy $h \nu$ is as follows:

$$
m_{\mathrm{el}} \cdot \Delta E_{\mathrm{el}}=n_{\mathrm{vm}} \cdot \Delta E_{\mathrm{vm}}+h \nu \approx n_{\mathrm{vm}} \cdot \Delta E_{\mathrm{vm}} .
$$

Here, $m_{\mathrm{el}}$ is the number of steps (gaps) between the quantized electron levels, and $n_{\mathrm{vm}}$ the number of vibration quanta helping a RF photon to get absorbed. According to the Kubo's formula (Kubo, 1962 , 1977) - see also Eq. A44 in the Appendix A. - the step in the electron energy levels $\Delta E_{\text {el }}$ depends on the number of gold atoms $N_{a}$. The condition (Eq. 5 ) that $m_{\mathrm{el}}$ energy steps must embrace $n_{\mathrm{vm}}$ phonon energies takes the form

$$
\frac{4}{3} m_{\mathrm{el}} \frac{E_{\mathrm{F}}}{N_{a}} \approx \frac{n_{\mathrm{vm}} v_{\mathrm{L}} h}{\pi(D-2 \delta)},
$$

where $L=\pi(D-2 \delta)$ is the length of the closed contour at the depth $\delta$ under the GNP surface, and the (longitudinal) sound velocity $v_{\mathrm{L}}$ relates frequency to wave vector. Further on, assuming that the density of gold in the surface shell and the transition shell is close to that in the core region, i.e. in bulk gold, the number of atoms in GNP can be expressed via density of gold $\rho$, atomic mass $m_{a}$ and the volume $V$ (or diameter $D$ ) of the particle:

$$
N_{a} \approx \frac{\rho V}{m_{a}}=\frac{\pi}{6} \frac{\rho}{m_{a}} D^{3} .
$$

Taken together with Eq. [6), this yields the depressed cubic equation on $D$ :

$$
\begin{gathered}
D^{3}+\mathfrak{p} D+\mathfrak{q}=0 \\
\text { with } \mathfrak{p}=-\frac{8 E_{\mathrm{F}} m_{a}}{v_{\mathrm{L}} h \rho}\left(\frac{m_{\mathrm{el}}}{n_{\mathrm{vm}}}\right) ; \quad \mathfrak{q}=\frac{16 E_{\mathrm{F}} m_{a} \delta}{v_{\mathrm{L}} h \rho}\left(\frac{m_{\mathrm{el}}}{n_{\mathrm{vm}}}\right) .
\end{gathered}
$$

For $\mathfrak{p}<0$ and $\mathfrak{q}>0$, Eq. (8) always has one negative root and either two (possibly degenerate) or none positive ones. The two positive roots of Eq. (8) can be expressed as follows:

$$
D_{k}=-2 \sqrt{\frac{|\mathfrak{p}|}{3}} \cos \left\{\frac{1}{3} \arccos \left[\frac{\mathfrak{q}}{2}\left(\frac{3}{|\mathfrak{p}|}\right)^{\frac{3}{2}}\right]+\frac{2 \pi}{3} k\right\},
$$

where $k=1,2$. Note that the parameters $\mathfrak{p}$ and $\mathfrak{q}$ contain, along with constants depending on the properties of gold, the trial numbers $\delta, m_{\mathrm{el}}$ and $n_{\mathrm{vm}}$; recall that $\delta$ is the depth of propagation of LAVM, and $n_{\mathrm{vm}}$ is the number of vibration quanta matching within the electronic excitation. The solutions $D_{k}$ can be expressed in terms of $\delta, m_{\mathrm{el}}$ and $n_{\mathrm{vm}}$ as follows ( $D_{k}$ and $\delta$ measured in $\mathrm{nm}$ ):

$$
D_{k}=-8.64 \sqrt{\frac{m_{\mathrm{el}}}{n_{\mathrm{vm}}}} \cos \left[\frac{1}{3} \arccos \left(0.695 \cdot \delta \sqrt{\frac{n_{\mathrm{vm}}}{m_{\mathrm{el}}}}\right)+\frac{2 \pi k}{3}\right] .
$$

Of two positive roots given by Eq. [11), we retain the practically relevant largest value of $D_{k}$, corresponding to $k=1$. Note that certain combinations $\left(m_{\mathrm{el}}, n_{\mathrm{vm}}\right)$ yield the argument of arccosine $>1$ and hence no solution. The roots are densely yet unevenly distributed, as is shown by Fig. 99. Note that the total number of solutions increases, as expected, with the number of trial $\left(m_{\mathrm{el}}, n_{\mathrm{vm}}\right)$ combinations, however the profile of the root density, with its narrow maximum around $\approx 7 \mathrm{~nm}$, remains stable up to the upper $\sim 25 \%$ of the total span of $D$ values. For this reason, and moreover since a non-ideal equidistanteness of both electrons' and LAVMs' levels would eventually "detune" the criteria set by Eqs. (5. 6) for large $\left(m_{\mathrm{el}}, n_{\mathrm{vm}}\right)$, we set, from now on, an arbitrary limit $(=10)$ on the maximal values of the latter.

We'll see below that this remarkable "selectivity" of GNP sizes $D$ with respect to their capacity to satisfy the energy conservation relations will eventually manifest itself in the distribution of the HR. The latter 


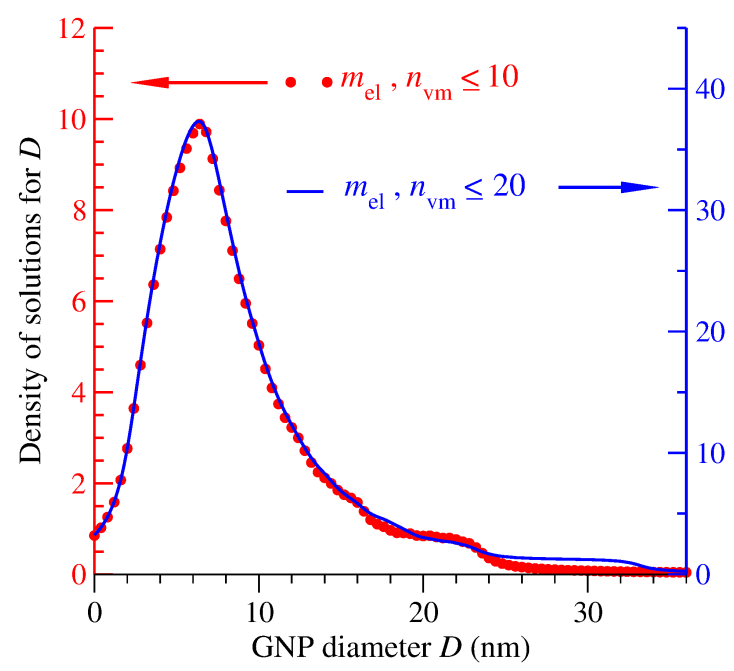

FIG. 9 The density of discrete solutions $D_{k=1}$ after Eq. (11), smeared into a continuous distribution with the half-width parameter of $1 \mathrm{~nm}$, for better visibility. $\delta$ is fixed to $0.7 \mathrm{~nm}$. The cases $\left(m_{\mathrm{el}}, n_{\mathrm{vm}}\right) \leq 10$ (red dots, refer to the left scale) and $\left(m_{\mathrm{el}}, n_{\mathrm{vm}}\right) \leq 20$ (blue continuous line, refer to the right scale) are compared. See text for details.

ought to be influenced, however, by the next element entering our discussion, namely, the availability of "useful" LAVMs that can be induced in the particles of selected sizes. "Useful" means the modes whose energies are multiples of $n_{\mathrm{vm}} \cdot \Delta E_{\mathrm{vm}}=n_{\mathrm{vm}} \cdot v_{\mathrm{L}} h /[\pi(D-2 \delta)], n_{\mathrm{vm}}$ being selected by the commensurability of the vibration energies with electron excitation ones, in the spirit of Eq. (5). Obviously, for the optimal heating it is essential to find many such vibrational modes within the FWHM of the LAVM-related VDOS peak of gold (cf. Figs. 78 and the related discussion), the number we'll refer to as $N_{\mathrm{FwHM}}$ in the following.

\section{B. Momentum conservation}

In addition to the energy conservation equation (5), one should take into account the momentum conservation condition. We'll specify it for the case of LAVM propagating along the circular contour of the diameter $D-2 \delta$, to which the phonon momentum $n_{\mathrm{vm}} \vec{q}$ is tangential - see Fig. 10 . Anywhere on the contour, a Fermi electron with the momentum $\vec{p}_{\mathrm{F}}$ can intervene to bring about an absorption of a RF photon (we neglect the effect of the momentum and energy of the latter onto the resulting conservation relation, as was already argued before).

The situation arbitrarily shown in Fig. 10 assumes the momentum of the Fermi electron to point inwards the GNP; on absorbing a phonon, such electron would transverse the particle along the path $l$ and release the phonon "on the other side" of the contour. A different possibility would be the $\vec{p}_{\mathrm{F}}$ pointing outwards; on the nanoparticle's surface such electron would be either elastically reflected inwards, or emit a quantum and be "lost" for the GNP heating mechanism we discuss. The probability relation for such elastic / inelastic

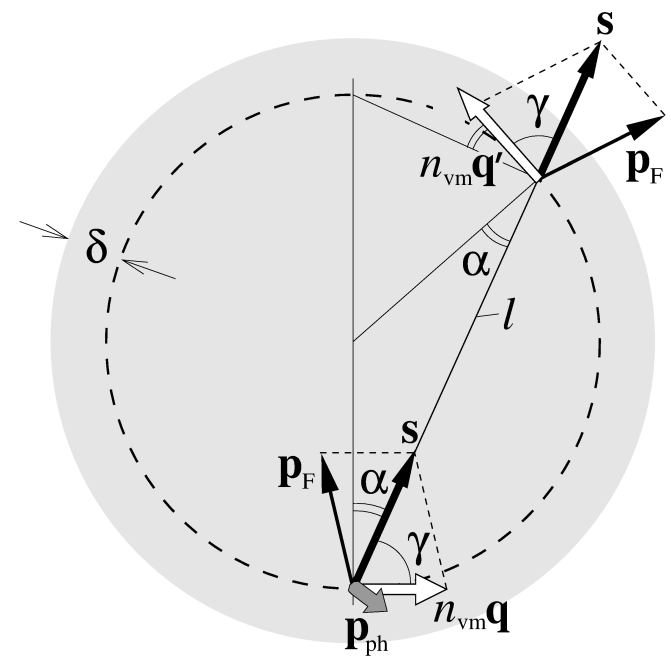

FIG. 10 Scheme of RF photon absorption on a circular contour inside a nanoparticle; see text for discussion. 
events at the surface is a priori difficult to estimate. Anyway, the elastically scattered electron will almost "instantly" regain the contour and follow the path of the inwards-moving electron, as depicted in Fig. 10 To specify what "instantly" means, we note that the perturbation of the potential inside the particle, "felt" by an electron, propagates, at most, with the longitudinal sound velocity in gold, i.e., $v_{\mathrm{L}}=3.23 \cdot 10^{5} \mathrm{~cm} \cdot \mathrm{s}^{-1}$, whereas the Fermi velocity in gold is three orders of magnitude larger: the free-electron model with the electron density corresponding to that of monovalent gold yields $v_{\mathrm{F}}=1.4 \cdot 10^{8} \mathrm{~cm} \cdot \mathrm{s}^{-1}$ (cf. Sec. III see also Ashcroft and Mermin, 1976). The "flight time" for the electron to reach the surface of the nanoparticle and get back to the contour is therefore $\sim 10^{-15} \mathrm{~s}$.

A simple geometric argument illustrated by Fig. 10 (for the ideal case of planar circular contour) shows that the momentum of the absorbed phonon $n_{\mathrm{vm}} \vec{q}$ can "ride" the electron across the particle and be released on the other side of the contour, that we'll refer to as relaxation. In fact, simultaneously released are the Fermi momentum $\vec{p}_{\mathrm{F}}$ and the phonon momentum $n_{\mathrm{vm}} \vec{q}^{\prime}$, both at angles with their respective "preabsorption" values but exactly preserving the corresponding moduli, under the condition that the phonon is emitted along the contour at the electron's contact with the latter on arrival. Specifically in Fig. 10. the transferred momentum $\vec{s}$, a sum of the electron $\vec{p}_{\mathrm{F}}$ and phonon $n_{\mathrm{vm}} \vec{q}$ momenta are related to the electron emission angle $\gamma$ as follows:

$$
p_{\mathrm{F}}^{2}=s^{2}+\left(n_{\mathrm{vm}} \cdot q\right)^{2}-2 s\left(n_{\mathrm{vm}} \cdot q\right) \cos \gamma,
$$

i.e., the angle the momentum of the excited electron makes to the contour is

$$
\gamma=\arccos \frac{s^{2}+\left(n_{\mathrm{vm}} \cdot q\right)^{2}-p_{\mathrm{F}}^{2}}{2 s\left(n_{\mathrm{vm}} \cdot q\right)} .
$$

Fig. 10implies moreover that the momentum of a LAVM phonon, via the interaction with an electron, may be "reinforced" by that of RF photon. The "parallel" (along the contour) component of the latter is then added to $n_{\mathrm{vm}} \cdot \vec{q}$ and transferred (by mediation of the excited electron) to the relaxation point. The momentum of then released phonon is $n_{\mathrm{vm}} \cdot \vec{q}^{\prime}$, with $\left|\vec{q}^{\prime}\right|>|\vec{q}|$, even if the net increase of the phonon momentum is quite small, with respect to electron and phonon counterparts: $h \nu \ll \Delta E_{\mathrm{vm}} ; p_{\mathrm{ph}} \ll n q, p_{\mathrm{ph}} \ll p_{\mathrm{F}}$.

The magnitude of $s$ in Eq. $\sqrt{13}$ is $s=\sqrt{2 m\left(E_{\mathrm{F}}+n_{\mathrm{vm}} \Delta E_{\mathrm{vm}}\right)}$. On expressing the magnitude of the LAVM momentum $q$ and the energy step via the nanoparticle size $D$ and the velocity of (longitudinal) sound $v_{\mathrm{L}}$,

$$
q=h /[\pi(D-2 \delta)] ; \quad \Delta E_{\mathrm{vm}}=v_{\mathrm{L}} h /[\pi(D-2 \delta)],
$$

we arrive at an expression for $\gamma$ in terms of $n_{\mathrm{vm}}$ and $(D-2 \delta)$ :

$$
\gamma=\arccos \left\{\left[m v_{\mathrm{L}}+\frac{n_{\mathrm{vm}} h}{2 \pi(D-2 \delta)}\right]\left[p_{\mathrm{F}}^{2}+\frac{2 m n_{\mathrm{vm}} v_{\mathrm{L}} h}{\pi(D-2 \delta)}\right]^{-\frac{1}{2}}\right\} .
$$

An excited electron would traverse the nanoparticle (along the chord $l$ in Fig. 10) and release energy on entering the cyclic contour again, whereby a vibration mode with momentum $n_{\mathrm{vm}} \vec{q}^{\prime}$ would be induced. Technically this might happen as a consequence of an electric field being suddenly created at the "arrival point" of the electron on the contour; the Coulombic attraction of the ions would trigger the compression wave to run along the contour. The electron path (chord) is related to the nanoparticle parameters $n_{\mathrm{vm}}, D$, $\delta$ as follows:

$$
\begin{aligned}
l & =(D-2 \delta) \cos \left(\frac{\pi}{2}-\gamma\right) \\
& =(D-2 \delta) \sin \arccos \left\{\left[m v_{\mathrm{L}}+\frac{n_{\mathrm{vm}} h}{2 \pi(D-2 \delta)}\right]\left[p_{\mathrm{F}}^{2}+\frac{2 m n_{\mathrm{vm}} v_{\mathrm{L}} h}{\pi(D-2 \delta)}\right]^{-\frac{1}{2}}\right\}
\end{aligned}
$$

For relevant values of $n_{\mathrm{vm}}$ and $(D-2 \delta)$, the argument of arccosine varies within $\sim 0.006-0.187$. Consequently the sine in the above formula stays within $0.982-0.999$, i.e., $l \approx(D-2 \delta)$ with, at most, $\approx 2 \%$ accuracy, therefore the excited electron must transverse the nanoparticle roughly along the latter's axis. On neglecting the discreteness on the circle of LAVM propagation (of the $D-2 \delta$ diameter), each its point may serve as the "source" of the excited electron. The regions of absorption of RF photons in the nanoparticle are the rings of $(D-2 \delta)$ diameter. 


\section{Electron free path}

The probability for an electron to transverse the particle, i.e., to escape being scattered along the path of the length $l$ and to reach the "opposite" point on the cyclic contour, equals $\exp \left(-l / l_{0}\right) \approx \exp \left[-(D-2 \delta) / l_{0}\right]$, where $l_{0}$ is the mean free path of an electron in the nanoparticle of the size $D$ (see below).

It seems logical that the $\mathrm{HR}$ of a nanoparticle of size $D$ is proportional to the following factors: $(i)$ the energy of absorbed RF photons, (ii) the length of the contour $\pi(D-2 \delta$ ) (as the absorption may occur in any point thereupon); (iii) the summary number $\sum_{i} N_{\text {FWHM }}^{(i)}$ of matching opportunities for the multiple energy step $n_{\mathrm{vm}} \cdot \Delta E_{\mathrm{vm}}$ to fall within the energy range of FWHM vibration modes [the estimates are $14.60-18.40 \mathrm{meV}$ according to (Muñoz et al., 2013), or $12.86-16.54 \mathrm{meV}$ according to D.Y. (Sun et al. 2001$)] 2^{2}$ and $(i v)$ the probability for an excited electron to undergo relaxation on the contour, i.e., $\exp \left[-(D-2 \delta) / l_{0}\right]$ :

$$
\mathrm{HR} \sim h \nu \cdot 2 \pi(D-2 \delta) \sum_{i=1} N_{\mathrm{FWHM}}^{(i)} \exp \left[-(D-2 \delta) / l_{0}\right]
$$

Here, the factor 2 accounts for two senses of propagation of vibration modes along the closed contour, and the index $i$, without further elaborating for the moment, identifies absorption / relaxation "events" likely to contribute to the HR. This summation should, at least, take into account different combinations $\left(m_{\mathrm{el}}, n_{\mathrm{vm}}\right)$, within the global limitation $\leq 10$ imposed on these parameters, which retain $m_{\mathrm{el}} / n_{\mathrm{vm}}$ and thus correspond to the same solution $D$ of Eq. (11), but whose values $N_{\mathrm{FWHM}}$ are different. In the next subsection, we'll adress an issue of multiple contours which may participate in the absorption and the relaxation events, in which relation the summation will be further explained. For the time being, we retain the general structure of the expected expression for the HR, and try to specify the relevant value of $l_{0}$. The upper limit on it is the mean free path in bulk gold, $\approx 35 \mathrm{~nm}$; in nanoparticles, due to irregularities of internal structure, $l_{0}$ ought to be much shorter. A priori, the smaller the size, the more likely the crystal lattice is distorted, hence the smaller the mean free path. Estimates for some "working" value for small enough GNP sizes, which were covered by the study by (Moran et al., 2009), can be gained from their experimental data reproduced in Fig. 1. with the help of insight given by Eq. (17).

Fig. 1 reveals a tendency towards the "saturation" of curves with an increase of the gold volume fraction. This reflects the loss of efficiency of heating the nanoparticles following their aggregation: the higher the GNP concentration, the higher the probability of their agglutination. Therefore the "net" $l_{0}$ values are more safely to extract from the slope of curves $d(\mathrm{HR}) / d($ volume fraction) near the origin. Of interest for us is the value of $l_{0}$ for GNPs with $D_{1}=5 \mathrm{~nm}$ and $D_{2}=10 \mathrm{~nm}$; the corresponding slopes are almost identical. Eq. (17) yields the following relation for the curves corresponding to these values of $D$ :

$$
\frac{\mathrm{HR}_{2}}{\mathrm{HR}_{1}} \approx \frac{D_{2}-2 \delta}{D_{1}-2 \delta} \cdot \frac{\sum_{i}^{\left(D_{2}\right)} N_{\mathrm{FWHM}}^{(i)}}{\sum_{j}^{\left(D_{1}\right)} N_{\mathrm{FWHM}}^{(j)}} \exp \left(\frac{D_{1}-D_{2}}{l_{0}}\right) .
$$

As mentioned above, each sum implies all relevant absorption / relaxation events within the particle of the corresponding size. Practical calculations show that, in order to estimate the $\mathrm{HR}_{2} / \mathrm{HR}_{1}$ ratio from the data of Fig. 1 with the accuracy of $\approx 10 \%$, it suffices to retain in Eq. 18 the leading term of each sum. Then, for the range of diameters $\simeq 5-10 \mathrm{~nm}, l_{0}$ can be expressed as follows:

$$
l_{0}=\left(D_{1}-D_{2}\right)\left[\ln \left(\frac{\mathrm{HR}_{2}}{\mathrm{HR}_{1}} \cdot \frac{D_{1}-2 \delta}{D_{2}-2 \delta} \cdot \frac{N_{\mathrm{FWHM}}^{(1)}}{N_{\mathrm{FWHM}}^{(2)}}\right)\right]^{-1} .
$$

The effective identity of the $d(\mathrm{HR}) / d$ (volume fraction) slopes characterizing the GNPs of $D_{1}=5 \mathrm{~nm}$ and $D_{2}=10 \mathrm{~nm}$ implies $\mathrm{HR}_{2} / \mathrm{HR}_{1} \approx 1 \mathrm{in} \mathrm{Eq.} 18$. For the contour $\delta=0.7 \mathrm{~nm}$ deep, $\left(D_{1}-2 \delta\right) /\left(D_{2}-2 \delta\right) \approx 0.42$. The relation $N_{\mathrm{FWHM}}^{(1)} / N_{\mathrm{FWHM}}^{(2)}$ follows from the straightforward counting of LAVMs that make a discrete spectrum on a circuar contour of the $(D-2 \delta)$ size: how many of modes will fall within the FWHM of the longitudinal acoustic peak of gold. In principle, $N_{\text {FWHM }}$ steadily increases with size, but the subtlety is that

\footnotetext{
${ }^{2}$ The sum $\sum_{i} N_{\text {FWHM }}^{(i)}$ takes into consideration contributions from the absorption of RF photons at phononic momenta $\mathbf{q}, 2 \mathbf{q}, i \mathbf{q}$ etc., in the spirit of Fig. 5 for fixed $m_{\mathrm{el}} / n_{\mathrm{vm}}$ and $\delta$.
} 
not all steps in vibration energy are compatible with the energy conservation criteria Eq. (5. 6). Appendix B explains this situation and argues that $N_{\mathrm{FWHM}}^{(1)}=1, N_{\mathrm{FWHM}}^{(2)} \approx 7.5$, hence $N_{\mathrm{FWHM}}^{(1)} / N_{\mathrm{FWHM}}^{(2)} \approx 0.13$, and from Eq. (19) $l_{0} \approx-5 \mathrm{~nm} / \ln (0.055)=1.72 \mathrm{~nm}$.

Coming back to the discussion at this subsection's opening, we can anticipate that the final HR, as function of GNP size, will be the interplay (multiplication) of three tendencies. The first one is the availablilty of "good" $D$ values which can contribute at all; they group around $\approx 7 \mathrm{~nm}$ and rapidly become scarse at larger sizes. The second tendency is the number of LAVMs within the "good" energy interval (given by the elastic properties of gold); this number essentially grows with $D$. The third effect is an exponential cutting of the HR at the characteristic length much shorter than the electron mean free path in the bulk gold. In total, the last tendency shifts the maximum of $\operatorname{HR}(D)$ a bit more to the left from the abovementioned "primary" $\approx 7 \mathrm{~nm}$ value than the second tendency shifts it to the right.

One can infer that, would the data for $D<5 \mathrm{~nm}$ be available in Fig. 1, one could expect the initial slopes of the corresponding curves (of HR vs gold volume fraction) to go steeper. Correspondingly, the $l_{0}$ values for such sizes would likely be less than $1.72 \mathrm{~nm}$, and, on the scale of the GNP sizes, the maximum of the HR would occur at slightly smaller values than so far reported.

We'll see in the following how the final counting of HRs proceeds, which also takes into account a somehow delicate issue of possible "diversification" of the absorption / relaxation events.

\section{Case of multiple contours}

At a risk of attributing too much precision to a simple enough model, we would like to emphasize a possibility for the energy of excited electron to be returned to a "different" phonon than that it was originally borrowed from. Once $N_{a}$ and $m_{\mathrm{el}}$ are fixed for a GNP, the Eq. 6) can be, in principle, satisfied for various values of $\delta^{\prime}>\delta$, each $\delta^{\prime}$ being a different "depth" of a circular contour, assuming for simplicity a symmetric placement of the latter within the particle. The phonon energy at each contour is quantified, so that

$$
\left(D-2 \delta^{\prime}\right) /(D-2 \delta)=n_{\mathrm{vm}}^{\prime} / n_{\mathrm{vm}},
$$

for integer $n_{\mathrm{vm}}^{\prime}$. Obviously, the case $n_{\mathrm{vm}}=1$ does not permit any inner contour, whereas $n_{\mathrm{vm}}>1$ allows $n_{\mathrm{vm}}^{\prime}=1, \ldots, n_{\mathrm{vm}}-1$. This is depicted in Fig. 11 for $n_{\mathrm{vm}}=1,2,3$, and summarized in Table II. In this way, the discretized phonon momentum values (integer) $\times h / L$ and the corresponding (momentum) $\times v_{\mathrm{L}}$ energies must find their "resonance" counterparts on relaxation. This is possible if the "secondary" contour length is commensurate with the "primary" one. The relation is not exact, since the energy absorbed by an electron is that of phonon(s) plus (much smaller) RF quantum, whereas the "relaxation" energy (released by the electron) is just the phonon(s)' one. However, it is helpful for counting different contributions. An essential observation is that the density of "resonance" modes decreases as the contour length shrinks. Still, the contributions from "inner" contours is not negligible; counting them, with different $n_{\mathrm{vm}} / n_{\mathrm{vm}}^{\prime}$ integer taken into account, would modify somehow the contributions of different GNP sizes to the HR.

For each "event", the "free path" of an excited electron $l_{i}$ prior to its relaxation can be easily calculated in analogy to how it is shown, for several selected $\left(n_{\mathrm{vm}}, n_{\mathrm{vm}}^{\prime}\right)$ combinations, in the last column of Table II]

TABLE II Characteristics of internal contours and the electron path transversing them for several small values of $n_{\mathrm{vm}}$. See text for details and refer to Fig. 11 .

\begin{tabular}{cccc}
\hline$n_{\mathrm{vm}}$ & $n_{\mathrm{vm}}^{\prime}$ & $\left(D-2 \delta^{\prime}\right) /(D-2 \delta)$ & $l /(D-2 \delta)$ \\
\hline 2 & 1 & $1 / 2$ & $1 / 4^{a} ; 3 / 4^{b}$ \\
3 & 1 & $1 / 3$ & $1 / 3^{c} ; 2 / 3^{d}$ \\
3 & 2 & $2 / 3$ & $1 / 6^{e} ; 5 / 6^{f}$ \\
4 & 1 & $1 / 4$ & $3 / 8 ; 5 / 8$ \\
4 & 2 & $2 / 4$ & $2 / 8 ; 6 / 8$ \\
4 & 3 & $3 / 4$ & $1 / 8 ; 7 / 8$ \\
\hline
\end{tabular}

Fig. 11 middle panel: ${ }^{a} A B,{ }^{b} A C$. Fig. 11, right panel: ${ }^{c} A C,{ }^{d} A D,{ }^{e} A B,{ }^{f} A E$. 

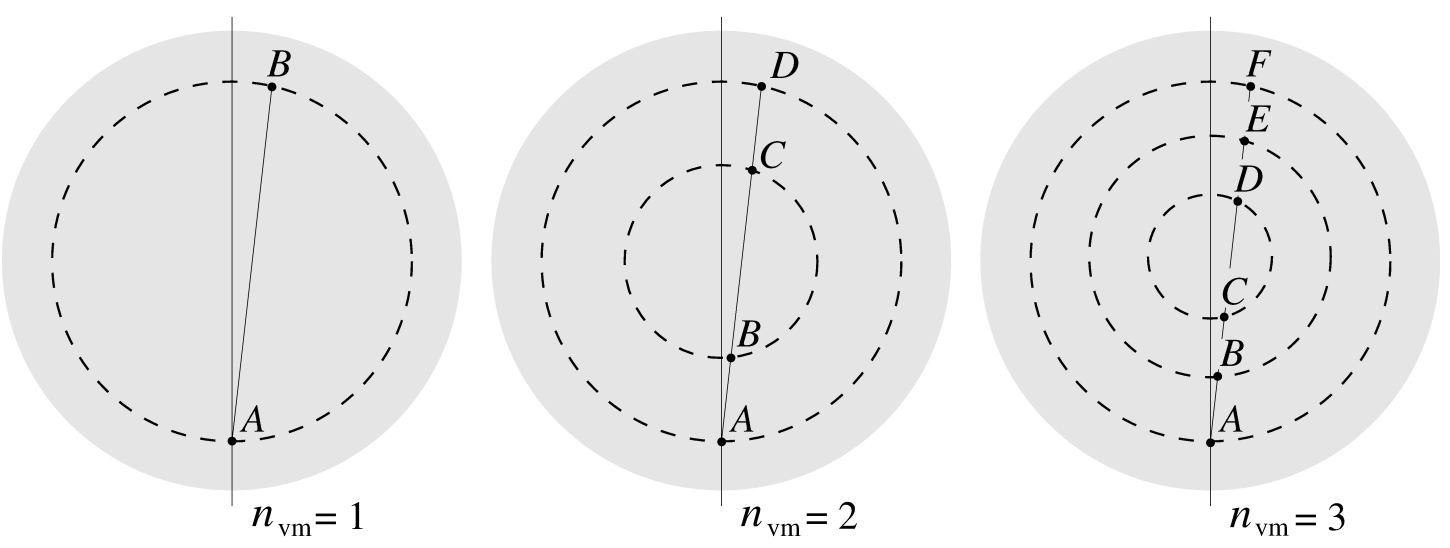

FIG. 11 Schematically drawn cyclic contours within nanoparticles for three different values of the $n_{\mathrm{vm}}$ parameter. " $A$ " marks the excitation site for a Fermi electron on the outer contour, following the absorption of a RF photon and the LAVM. Other points indicate possible sites of relaxation of the excited electron on traversing the particle.

in terms of the contour size. The values are listed in pairs, indicating that, as the electron traverses the GNP approximately along the diameter (by force of earlier envoked arguments), its "exit point" may occur on a close or on a remote point on a given internal contour.

Alternatively, a situation is imaginable that the resonance criteria of Eqs. (5, 6) allow a modification of electron energy step on exchange with a phonon, on respecting the condition

$$
\frac{\nu h}{m_{\mathrm{el}} \cdot \pi(D-2 \delta)}=\frac{\nu h}{m_{\mathrm{el}}^{\prime} \cdot \pi\left(D-2 \delta^{\prime}\right)}, \quad \text { hence } \quad \frac{D-2 \delta^{\prime}}{D-2 \delta}=\frac{m_{\mathrm{el}}}{m_{\mathrm{el}}^{\prime}} .
$$

Since the last relaton is $<1$ (the secondary contour at depth $\delta^{\prime}$ is deeper than the primary one), $m_{\mathrm{el}}^{\prime}>m_{\mathrm{el}}$; moreover, $m_{\mathrm{el}}^{\prime} / m_{\mathrm{el}}$ must be an integer.

Possible contributions to the HR, in the spirit of Eq. (17), are summarized in Table III and depicted in Fig. 12. The summation in the fourth column of Table III selects the cases ( $m_{\mathrm{el}}$ unchanged, $n_{\mathrm{vm}}^{\prime}$ variable) while that in the fifth column - the cases ( $m_{\mathrm{el}}^{\prime}$ variable, $n_{\mathrm{vm}}$ unchanged).

The condition $m_{\mathrm{el}}^{\prime} \leq 10$ combined with that of $m_{\mathrm{el}}^{\prime} / m_{\mathrm{el}}$ to be integer does considerably restrict the amount of inner contours; hence so few non-zero entries in the fifth column of Table III. The largest contributions (for $D=6.65$ and $9.80 \mathrm{~nm}$ ) come out because, with their small initial $m_{\mathrm{el}}$ value, the largest number of contours $(=4)$ could be generated. The summation over possible contours and transitions yields a global

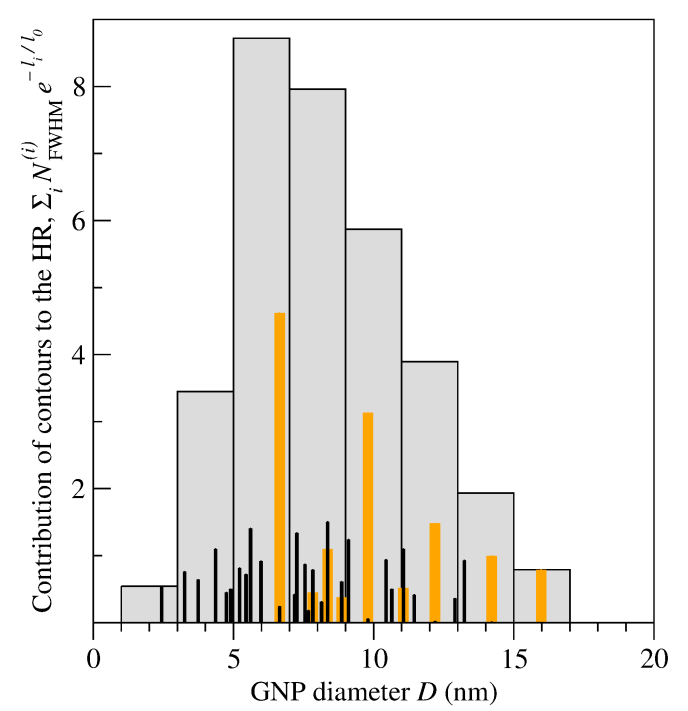

FIG. 12 Contributions of possible contours in GNPs of different sizes to their HR. Vertical lines mark the "individual" values of $N_{\mathrm{FWHM}}^{(i)} \exp \left(-l_{i} / l_{0}\right)$ for each "valid" contour within a "resonant" GNP size, whereby thin black lines stand for contributions of the $\left(m_{\mathrm{el}}^{\prime}\right.$ variable, $n_{\mathrm{vm}}=$ const) type, and thick orange lines - for contributions of the $\left(m_{\mathrm{el}}=\right.$ const, $n_{\mathrm{vm}}^{\prime}$ variable) type. Light gray bars make a histogram of the abovementioned contributions, summed up within $2 \mathrm{~nm}$ steps of GNP diameter $D$. 
TABLE III Contributions to the HR from summations over primary and corresponding secondary contours characterized by different $\left(m_{\mathrm{el}}, n_{\mathrm{vm}}\right)$ values in gold nanoparticles of diameters $D$. See text for details.

\begin{tabular}{|c|c|c|c|c|}
\hline \multirow[b]{2}{*}{$m_{\mathrm{el}}$} & \multirow[b]{2}{*}{$n_{\mathrm{vm}}$} & \multirow[b]{2}{*}{$D(\mathrm{~nm})$} & \multicolumn{2}{|c|}{$\sum_{i} N_{\mathrm{FWHM}}^{(i)} \exp \left(-l_{i} / l_{0}\right)$} \\
\hline & & & $\sum_{i} \equiv \sum_{n_{\mathrm{vm}}^{\prime}}, n_{\mathrm{vm}}^{\prime} \leq 10$ & $\sum_{i} \equiv \sum_{m_{\mathrm{el}}^{\prime}}, m_{\mathrm{el}}<m_{\mathrm{el}}^{\prime} \leq 10$ \\
\hline 1 & 4 & 2.44 & 0.55 & 0 \\
\hline 1 & 3 & 3.26 & 0.76 & 0 \\
\hline 2 & 5 & 3.74 & 0.64 & 0 \\
\hline 1 & 2 & 4.36 & 1.10 & 0 \\
\hline 4 & 7 & 4.75 & 0.45 & 0 \\
\hline 3 & 5 & 4.90 & 0.50 & 0 \\
\hline 2 & 3 & 5.22 & 0.82 & 0 \\
\hline 5 & 7 & 5.45 & 0.72 & 0 \\
\hline 3 & 4 & 5.61 & 1.41 & 0 \\
\hline 5 & 6 & 5.98 & 0.92 & 0 \\
\hline 1 & 1 & 6.65 & 0.24 & 4.62 \\
\hline 8 & 7 & 7.18 & 0.42 & 0 \\
\hline 7 & 6 & 7.26 & 1.34 & 0 \\
\hline 5 & 4 & 7.55 & 0.87 & 0 \\
\hline 9 & 7 & 7.67 & 0.18 & 0 \\
\hline 4 & 3 & 7.83 & 0.79 & 0.45 \\
\hline 10 & 7 & 8.14 & 0.31 & 0 \\
\hline 3 & 2 & 8.36 & 1.51 & 1.10 \\
\hline 5 & 3 & 8.86 & 0.61 & 0.38 \\
\hline 7 & 4 & 9.11 & 1.24 & 0 \\
\hline 2 & 1 & 9.80 & 0.06 & 3.13 \\
\hline 9 & 4 & 10.44 & 0.94 & 0 \\
\hline 7 & 3 & 10.65 & 0.50 & 0 \\
\hline 5 & 2 & 11.06 & 1.10 & 0.52 \\
\hline 8 & 3 & 11.45 & 0.42 & 0 \\
\hline 3 & 1 & 12.19 & 0.02 & 1.48 \\
\hline 10 & 3 & 12.90 & 0.36 & 0 \\
\hline 7 & 2 & 13.24 & 0.93 & 0 \\
\hline 4 & 1 & 14.21 & 0.01 & 1.00 \\
\hline 5 & 1 & 15.98 & 0 & 0.79 \\
\hline 6 & 1 & 17.58 & 0 & 0 \\
\hline 7 & 1 & 19.06 & 0 & 0 \\
\hline 8 & 1 & 20.43 & 0 & 0 \\
\hline 9 & 1 & 21.71 & 0 & 0 \\
\hline 10 & 1 & 22.93 & 0 & 0 \\
\hline
\end{tabular}

scan of the property $\sum_{i} N_{\mathrm{FWHM}}^{(i)} \exp \left(-l_{i} / l_{0}\right)$ which is expected to be proportional to the HR, as function of the GNP diameter. The results are shown in Fig. 12 in two ways: as a contribution (weighted by $e^{-l_{i} / l_{0}}$ ) of each relaxation event, possible in a GNP of given size, and as cumulate effect of multiple events, grouped in a histogram with a fixed step in $D$. The first representation indicates that the contributions start from $D=2.44 \mathrm{~nm}$ and drop down to zero beyond $D=15.98 \mathrm{~nm}$. The histogram representation seems more helpful in inspecting the "importance" of different GNP sizes for the HR. It seems that the HR efficiency 
peaks around $D \approx 6 \mathrm{~nm}$ and rapidly decreases for smaller and larger diameters.

To conclude the discussion about multiple contours, we point out that the excitation energy for an electron can also be borrowed from LAVM propagating along one of internal contours. However, the probability of such effects rapidly decreases with descending onto ever shorter contours characterized by ever sparser distribution of quantized resonance modes.

\section{ON THE REDUCED HEAT PRODUCTION IN AGGREGATED GNPS}

An aggregation of $n$ GNPs yields a larger particle, with the number of atoms and electrons increased by the factor of $n$. However, this won't be normally accompanied by a formation of joint subsurface contour at the depth $\delta$, as the core regions of different GNPs remain isolated from each other by their surface shells. Therefore the "optimal" conditions of RF photon absorption, given by Eqs. (5) and (6), would be violated, and the HR of aggregated GNPs reduced.

After Fig. 1, the HR eventually saturates, for all particle sizes, as function of gold volume fraction. For small GNPs this saturation occurs faster, for the apparent reason that, for a given gold volume fraction, smaller particles mean their higher concentration, and hence higher tendency for aggregation.

\section{FURTHER SUGGESTIONS FOR ENHANCING HEATING RATES IN GNPS}

In Sec. III it has been shown that in GNPs, the HR is enhanced thanks to large number of participating LAVMs. As heating of GNPs involves both the LAVMs and the Fermi electrons, one way of enhancing the $\mathrm{HR}$ ever further would be to increase the number of electrons absorbing the RF photons. This is possible through enhancing the local electronic DOS (EDOS) at the Fermi energy of gold, doping the latter with transition metal impurities, e.g., Ta or Fe - see Fig. 13, and also (Moldosanov and Postnikov, 2013, Postnikov and Moldosanov, 2012). The main peaks of spin-split local EDOS of these impurities, according to firstprinciples calculations ${ }^{3}$ are pinned at the Fermi level of gold, adding substantially to the bulk EDOS of the latter ${ }^{4}$ In addition to just increasing the number of electrons absorbing the RF photons, such doping would also enhance the scattering intensity of excited electrons. Both of these tendencies are expected to result in enhanced HR.

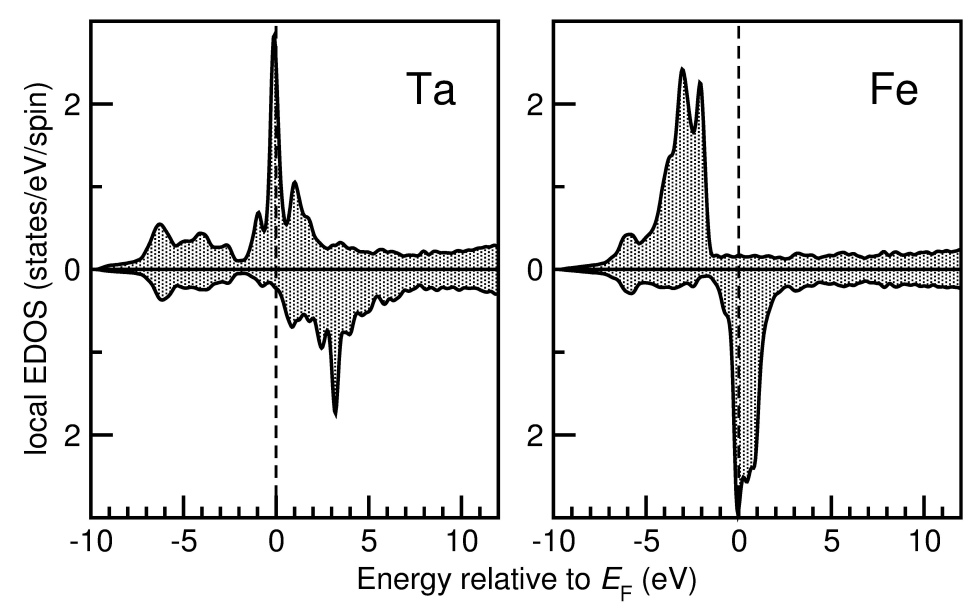

FIG. 13 Calculated spin-resolved local EDOSs against energy relative to $E_{\mathrm{F}}$ for Ta and Fe impurities in $\mathrm{Au}$, reproduced from (Postnikov and Moldosanov, 2012). Calculated EDOS of (Muñoz et al., 2013 ) for Fe $d$-electrons in 32-atom quasirandom structure of $\mathrm{Au}_{30} \mathrm{Fe}_{2}$ supports our results for Fe impurities in $\mathrm{Au}$ (Postnikov and Moldosanov, 2012).

\footnotetext{
${ }^{3}$ Our calculations were done within the density functional theory, using the generalised gradient approximation for the exchange-correlation, by the SIESTA method (see http://departments.icmab.es/leem/siesta/), allowing full atom relaxation for the 64 -atom supercell $(4 \times 4 \times 4$-replicated fcc primitive cell with one atom substituted by impurity).

${ }^{4}$ The calculations done on 64-atom super cells (one impurity of Ta or Fe per $63 \mathrm{Au}$ atoms) indicate that the total EDOS at the Fermi level is increased by $\sim 50 \%$ compared to the pure Au.
} 
Another imaginable way to bring about high EDOS would be due to $f$-electronic states residing at the Fermi level, that is the case of heavy-fermion compounds - see (Stewart, 1984) for a review. Such compounds, typically possessing a rare-earth element as their essential constituent, exhibit an anomalously high residual resistivity at low temperatures, traceable to high electron scattering intensity, and, in its turn, - to a strong peak in the density of states of $f$ electrons at the Fermi level. The presence of the compound $\mathrm{CeCu}_{6}$ among heavy-fermion systems permits to presume that gold, like a homologue of copper, may host $f$-element atoms so as to give rise to heavy-fermion behaviour. The nanoparticles of such compounds might possess a yet elevated heating rate.

\section{DISCUSSION}

The analysis of conservation conditions for energy $\left(m_{\mathrm{el}} \cdot \Delta E_{\mathrm{el}} \approx n_{\mathrm{vm}} \cdot \Delta E_{\mathrm{vm}}\right)$ and momentum (i.e., for the movement direction of the excited electron after absorption of the RF photon and the LAVM) lets estimate the size of GNPs capable to produce high HR via a simultaneous involvement of several vibration modes with energies matching $n_{\mathrm{vm}} \cdot \Delta E_{\mathrm{vm}}$, situated within the LAVMs of gold. It follows from our estimates that the optimal size of GNPs to use in RF hyperthermia is $5-7 \mathrm{~nm}$.

A number of known results seem to support our argumentation. (Moran et al. 2009), who explored heating of GNPs with sizes from 5 to $250 \mathrm{~nm}$ in the electric field of $13.56 \mathrm{MHz}$ frequency, observed that the highest HR was inherent to GNPs of $5 \mathrm{~nm}$ size. (Kruse et al., 2011) heated the GNPs by the $13.56 \mathrm{MHz}$ electric field too, and, again, the smallest tested GNPs $(\approx 5 \mathrm{~nm}$ size $)$ revealed the maximum HR.

Other researches testify that as the GNPs sizes deviate from $5-7 \mathrm{~nm}$, the HRs change from bad to worse. (Murdock and Hussain, 2010) observed the heating of GNPs with the $4 \mathrm{~nm}$ and $13 \mathrm{~nm}$ diameters and pointed out that their HRs were several times lower than those for the $5 \mathrm{~nm}$ GNPs in the work by (Moran et al. 2009). (Li et al., 2011) exposed the GNPs with sizes of 20, 50 and $200 \mathrm{~nm}$ to the $13.56 \mathrm{MHz}$ electric field and did not observe heating. In view of our analysis, the GNPs of 20 or $50 \mathrm{~nm}$ fall short of sizes (shown in Table I that would enable a "direct" absorption of a RF photon; at the same time, these sizes are much larger than 5-7 nm, the "optimum" diameter for the phonon-assisted absorption. As for the $200 \mathrm{~nm}$ size, that falls close to a number appearing in Table I] one can presume that the resonance for direct absorption is quite sharp and not satisfied in the real GNPs studied.

(Liu et al. 2012) did not detect any significant heating of GNPs with sizes of 15 to $30 \mathrm{~nm}$ (i.e. larger than the optimum size) at the $13.56 \mathrm{MHz}$ frequency. (Sirotkina et al. 2012 ) tried to treat tumours by the $\mathrm{RF}$ radiation of frequency $2.45 \mathrm{GHz}$ using the gold nanorods of $30 \mathrm{~nm}$ diameter and $60 \mathrm{~nm}$ length, whereby no anticancer effect was identified. In our opinion, namely the large sizes of nanorods were the problem.

(Corr et al. 2012a) and (Raoof et al. 2012) pointed out that heat generation was significantly reduced when the GNPs were aggregated. We attribute it to the fact that, as the GNPs come in contact, the common size of the formed aggregate is increased, and the condition 5 is not anymore fulfilled, resulting in reduction of HRs of GNPs.

A valuable contribution to the analysis of experimental and theoretical studies in heating kinetics of GNPs in the RF range was offered by a recent work by (Letfullin et al. , 2015). The authors have noted a spectacular failure of previous theoretical conclusions to account for the experimental evidence that RF radiation is capable of heating the GNPs. Our present work that emphasises the crucial role of LAVMs in the RF absorption suggests a plausible explanation of the above problem.

(Huang et al. 2012) who studied in vivo the size dependence (within the 2 to $15 \mathrm{~nm}$ range) of the GNPs' localization and penetration in cancer cells, multicellular spheroids and tumours, concluded that the 2 and $6 \mathrm{~nm}$ GNPs demonstrated advantages over larger nanoparticles in terms of tumour uptake and permeability. Such GNPs were able to penetrate deeply into tumour tissue and provide high levels of accumulation in it. As an application of the smallest ( $\sim 2 \mathrm{~nm}$ size) GNPs seems questionable due to loosening their biocompatibility (Leifert et al. 2013, Pan et al., 2012, 2007), the 5 - $7 \mathrm{~nm}$ GNPs, possessing also the highest HRs, become very valuable instruments in the $\mathrm{RF}$ hyperthermia.

Throughout the whole range of frequencies $\nu$ used in medical or biological studies $(\nu \sim 10 \mathrm{MHz}-3 \mathrm{GHz})$, the RF photon energies $h \nu$ are small compared to $n_{\mathrm{vm}} \cdot \Delta E_{\mathrm{vm}}$ of Eq. (5), for the LAVMs energy range of $\approx 3$ $-21 \mathrm{meV}$. Consequently, the condition (5) is generally satisfied, and the GNPs of about $5-7$ nm size would have elevated HRs not only at $13.56 \mathrm{MHz}$, but everywhere within the mentioned interval of frequencies; in particular, at $2.45 \mathrm{GHz}$, the operation frequency of a domestic microwave oven with fine, inverter-regulated 
power control. The use of the latter for laboratory investigations would permit performing genetic and biological experiments in vivo - e.g., with GNPs immersed in the tissues of larvae of such classical research target as Drosophila melanogaster.

Recently, (San et al. 2013) reported that at the frequency of $0.35 \mathrm{MHz}$ the Pt nanoparticles possessed HR twice higher than that of the GNPs and proposed to use them in RF hyperthermia. We attribute high $\mathrm{HR}$ of $\mathrm{Pt}$ nanoparticles to enhanced EDOS at the Fermi level of Pt, as compared to Au. For this reason, in $\mathrm{Pt}$, a number of electrons able to absorb the RF photons is enhanced.

One should expect that in conditions of the experimental capacitive electric transfer system used by (San et al. , 2013) in nanoparticles of AuPd and AuPt alloys (Pd and Pt content within $40-70$ mass \%), the HRs would be even higher than those in Pt nanoparticles. This is because in these alloys, judging by their enhanced EDOS at $E_{\mathrm{F}}$, the intensity of scattering of Fermi electrons is higher than that in pure Pt. However, an application of $\mathrm{AuPd}$, AuPt, Pd and Pt nanoparticles in the RF hyperthermia is questionable due to their enhanced catalytic activities.

Compared with these nanoparticles, the GNPs containing impurities of Ta and/or Fe, and hence developing an enhanced EDOS at the Fermi level and, expectedly, an enhanced intensity of electron scattering, seem to be much more promising for the RF hyperthermia using the GNPs (Moldosanov and Postnikov, 2013; Postnikov and Moldosanov, 2012).

During last years, the use of GNPs as systems of drug and gene delivery into cancer cells has expanded considerably. An opportunity to synergistically combine these techniques with RF hyperthermia may open new terrains in contemporary cancer theranostics. Recently (Conde et al., 2015$)$, apparently driven by a need to sense and overcome the cancer multidrug resistance, invented an implantable hydrogel with embedded DNA-coated GNPs of $\sim 10-17 \mathrm{~nm}$ diameters. Our above discussion hints that an attempt to go towards slightly smaller particles, of $5-7 \mathrm{~nm}$ diameter, would "activate" the embedded GNPs for the RF hyperthermia, adding such an option to the GNPs' other functions.

Mirkin and co-workers (Chinen et al., 2015, Cutler et al., 2012, Jensen et al., 2013 , Kouri et al., 2015. Radovic-Moreno et al., 2015; Randeria et al. , 2015) developed and used spherical nucleic acid GNP conjugates (13 nm diameter gold cores functionalized with densely packed and highly oriented nucleic acids). Hypothetically, the $5-7 \mathrm{~nm}$ diameter GNPs along with optional RF heating thereof could be used in these cancer treating technologies, providing new research opportunities through temperature control.

Another issue that enters the domain of feasible is the transfer of technologies developed for the plasmonic heating of GNPs onto the RF range. Zharov and co-workers (Galanzha et al. 2009, Nedosekin et al., 2013), aiming to prevent metastasis, used magnetic trapping of tumour cells circulating in the bloodstream with their simultaneous photoacoustic and photothermal detection. To this end, the gold-plated carbon nanotubes were employed. As it seems, the use of the 5-7 nm diameter GNPs instead of gold-plated carbon nanotubes may help to extend this method over applications in the RF range as well.

A recent work by (Hembury et al. . 2015) describes a promising so-called "quantum rattle", that is, a hollow spherical particle ( $\sim 150 \mathrm{~nm}$ of total diameter $)$ with mesoporous silica shell ( $\sim 25 \mathrm{~nm}$ thickness $)$ hosting both gold quantum dots (AuQDs) of $<2 \mathrm{~nm}$ diameter and GNPs (average crystallite size $7.3 \mathrm{~nm}$ in diameter). The quantum rattle is highly biocompatible and combines both cancer imaging and tumor treatment capabilities (chemotherapy and photothermal therapy in near infrared range). All these advantages in matching and even outperforming the state-of-the-art nanotechnology-based medical agents are achieved thanks to just AuQDs, whereas a contribution of GNPs is very small. Specifically, the photothermal therapy using the quantum rattles is provided through excitation of AuQDs by an infrared laser, then the AuQDs emit infrared fluorescence and enough heat to kill cancerous cells. In these quantum rattles, the GNPs are likely just processing waste. Meanwhile, as their sizes $(7.3 \mathrm{~nm})$ happen to fall close to the "favourable" range of $5-7 \mathrm{~nm}$, the presence of GNPs in quantum rattles under discussion could be used for additional targeted heating of the latter by RF irradiation.

Another recent work by (Tsai et al., 2015 ) outlines the use of gold nanorods (of $10 \mathrm{~nm}$ diameter / $41 \mathrm{~nm}$ length) connected to nanodiamonds, whereby the gold part serves to hyperthermia, by near-infrared laser heating, and the nitrogen-vacancy centers in diamond serve for temperature sensing. Here again, the use of nanoparticles of $5-7 \mathrm{~nm}$ size in place of nanorods would hopefully help to extend this technique over RF-induced hyperthermia.

Last but not least, the RF heating of GNPs may be of interest for developers of therapeutic strategies targeting to inhibit amyloidogenic process in the Alzheimer's disease. (Araya et al., 2008) suggested to use in this context the GNPs of $12.5 \pm 1.7 \mathrm{~nm}$ size heated by the microwave $14 \mathrm{GHz}$ radiation. Chances are that 
using the GNPs of "optimal" sizes would be useful for these tasks, too.

\section{CONCLUSION}

We suggest a physical model of the size effect in heat generation in GNPs, which also accounts for a reduction of heat generation as the GNPs get aggregated. In this model, the LAVMs (dominating in the distribution of vibrational density of states) play an important role - an apparently novel element in the related theory framework. According to our model, the heating of GNPs is thought to consist of two consecutive processes: first, the Fermi electron absorbs simultaneously the RF photon and the LAVM available in the GNP; hereafter the excited electron is relaxed, exciting a LAVM with the energy higher than that of the previously absorbed LAVM. The model predicts that the GNPs to be effectively heated should possess diameters of $\sim 5-7 \mathrm{~nm}$, i.e., very close to the experimentally inspected $\sim 5 \mathrm{~nm}$. The absorption band is expected to be very wide $(\sim 10 \mathrm{MHz}-3 \mathrm{GHz})$. This allows the use of frequencies typical for the "conventional" RF hyperthermia (without conducting nanoparticles). The energy release in the GNPs can be optimized by tuning the RF frequency, searching a compromise between the HR energy transfer efficiency and a penetration depth of the RF radiation into the biological tissue. The GNPs containing Ta or Fe impurity atoms are expected to be more effective heaters compared to nanoparticles of pure gold, due to enhanced electron density of states at the Fermi level. Gold nanoparticles with rare-earth impurity atoms are also brought into consideration as promising for the RF hyperthermia with conducting nanoparticles. The significance of the present study follows from the fact that the cancer specialists seeking approval for human clinical trials on the basis of their experimental results remain thus far in the dark in what regards the physical mechanism behind the observed trends.

\section{ACKNOWLEDGMENTS}

The authors sincerely thank Drs. Curley, Muñoz and Gong for their kind permissions to use the figures from their works. We also would like to thank Drs. Muñoz and Kresch for useful discussions of their measurements of the phonon DOS in gold.

\section{Appendix A: Level spacing vs number of atoms in a GNP}

(Kubo, 1962, 1977) adopted the one-electron approximation to electrons in small metallic particles, whereupon D.Y. (Sun et al. 2001) predicted and J. (Sun et al. 2014) experimentally demonstrated the existence of the bulklike core region in them. This justifies considering the GNP as a crystalline one and permits to specify a relationship between the level spacing of electrons and number of atoms in GNP, using formulas for the free electron gas. A GNP of cuboid shape and consisting of $N_{a}$ atoms contains (one "free" electron per atom of gold) $N_{a}$ electrons filling the energy levels up to the Fermi energy $E_{\mathrm{F}}$. The free-electron EDOS is (e.g., Wert and Thomson, 1970):

$$
\rho(E)=d N^{\prime} / d E=4 \pi(2 m)^{3 / 2} L^{3} E^{1 / 2} h^{-3},
$$

where $d N^{\prime}$ is a number of states within the energy interval $d E, L$ is the cuboid's edge and $h$ the Planck constant. Then the total number of electrons is

$$
N_{a}=\int_{0}^{E_{\mathrm{F}}} \rho(E) d E=\frac{8 \pi}{3}\left(2 m E_{\mathrm{F}}\right)^{3 / 2}\left(\frac{L}{h}\right)^{3} .
$$

In accordance with Eq. (A1), the spacing $\Delta E_{\text {el }}$ between the Fermi level and nearest energy level above it equals $\Delta E_{\mathrm{el}} \approx \Delta N^{\prime} / \rho\left(E_{\mathrm{F}}\right)$, where $\Delta N^{\prime}=2$, hence

$$
\Delta E_{\mathrm{el}} \approx \frac{2}{\rho\left(E_{\mathrm{F}}\right)}=\frac{h^{3}}{2 \pi(2 m)^{3 / 2} L^{3} E_{\mathrm{F}}^{1 / 2}} .
$$


From Eqs. A2 and A3, the Kubo's formula is deduced:

$$
\Delta E_{\mathrm{el}} \approx \frac{4}{3} \frac{E_{\mathrm{F}}}{N_{a}}
$$

\section{Appendix B: Counting vibration modes in GNPs of given size, within given energy range}

Assuming that the LAVMs propagate with (longitudinal) sound velocity $v_{\mathrm{L}}$ along the circular contour at depth $\delta$ under the surface of spherical particle of diameter $D$, the phonon energy quantum is $\Delta E_{\mathrm{vm}}=$ $v_{\mathrm{L}} h /[\pi(D-2 \delta)]$. However, insofar as phonons are absorbed /created in the process of the electron excitations / relaxations, the phonon energies can only change in blocks, commensurable with the electron states quantization, i.e., respecting the energy conservation condition of Eq. (5), $m_{\mathrm{el}} \Delta E_{\mathrm{el}} \approx n_{\mathrm{vm}} \Delta E_{\mathrm{vm}}$. Only certain combinations $\left(m_{\mathrm{el}}, n_{\mathrm{vm}}\right)$ are possible, that selects the "resonant" values of $D$, as given by Eq. (11). Table III lists the allowed groups $\left(m_{\mathrm{el}}, n_{\mathrm{vm}}, D\right)$ in the increasing order of $D$.

Searching in Table III for the $D$ values closest to the "reference" ones $D_{1}=5 \mathrm{~nm}$ and $D_{2}=10 \mathrm{~nm}$, one finds $D=5.22 \mathrm{~nm}$ that comes along with $n_{\mathrm{vm}}=3$, and $D=9.80 \mathrm{~nm}$ that comes along with $n_{\mathrm{vm}}=1$. We note that the apparently "competitive" values of $D$ by their closeness to the reference values, namely, $D=4.90 \mathrm{~nm}$ and $10.44 \mathrm{~nm}$, can only be selected with much higher values of $n_{\mathrm{vm}}$ and, consequently, may only intervene with much sparser distribution, and hence much smaller impact, or their allowed vibration modes. For $D=5.22 \mathrm{~nm}, n_{\mathrm{vm}} \Delta E_{\mathrm{vm}}=3.35 \mathrm{meV}$, so there is no more than just one vibration mode, of the energy $5 \times\left(n_{\mathrm{vm}} \Delta E_{\mathrm{vm}}\right)$, that falls within the FWHM range of the interest, 14.6 to $18.4 \mathrm{meV}$ (see caption to Fig. 8 and the related text), hence $N_{\mathrm{FWHM}}^{(1)}=1$ in Eq. 19. . For $D=9.80 \mathrm{~nm},\left(n_{\mathrm{vm}}=1\right) \cdot \Delta E_{\mathrm{vm}}=0.51 \mathrm{meV}$, and the above cited FWHM range may hosts much more modes at multipliers of this energy, namely, $N_{\mathrm{FWHM}}^{(2)} \approx 7.5$ in Eq. (19).

\section{REFERENCES}

Araya, E., I. Olmedo, N. G. Bastus, S. Guerrero et al. (2008). Gold nanoparticles and microwave irradiation inhibit beta-amyloid amyloidogenesis. Nanoscale Res. Lett. 3(11), 435. URL http://www. nanoscalereslett.com/content/3/11/435.

Ashcroft, N. W. and N. D. Mermin (1976). Solid State Physics. Saunders College.

Chinen, A. B., C. M. Guan and C. A. Mirkin (2015). Spherical nucleic acid nanoparticle conjugates enhance G-quadruplex formation and increase serum protein interactions. Angew. Chem. Int. Ed. 54(2), 527.

Coleridge, P. T. and I. M. Templeton (1982). Fermi-surface radii in copper, silver, and gold. Phys. Rev. B: Condens. Matter 25, 7818. URL http://link.aps.org/doi/10.1103/PhysRevB.25.7818.

Conde, J., N. Oliva and N. Artzi (2015). Implantable hydrogel embedded dark-gold nanoswitch as a theranostic probe to sense and overcome cancer multidrug resistance. Proc. Natl. Acad. Sci. U.S.A. 112(11), E1278. URL http: //Www.pnas.org/content/112/11/E1278.abstract.

Corr, S. J., M. Raoof, Y. Mackeyev, S. Phounsavath et al. (2012a). Citrate-capped gold nanoparticle electrophoretic heat production in response to a time-varying radio-frequency electric field. J. Phys. Chem. C, Nanomater. Interfaces 116(45), 24380. URL http://dx.doi.org/10.1021/jp309053z.

Corr, S. J., M. Raoof, L. J. Wilson and S. A. Curley (2012b). Chapter 6: Nanoparticles for noninvasive radiofrequency-induced cancer hyperthermia. In: M. Hepel and C.-J. Zhong (editors), Functional Nanoparticles for Bioanalysis, Nanomedicine, and Bioelectronic Devices: Volume 2, ACS Symposium Series, 81-94. American Chemical Society. URL http://pubs.acs.org/doi/abs/10.1021/ bk-2012-1113. ch006.

Cutler, J. I., E. Auyeung and C. A. Mirkin (2012). Spherical nucleic acids. J. Am. Chem. Soc. 134(3), 1376. URL http://dx.doi.org/10.1021/ja209351u.

Dal Corso, A. (2013). Ab initio phonon dispersions of transition and noble metals: effects of the exchange and correlation functional. J. Phys. Condens. Matter 25(14), 145401. URL http://stacks.iop. org/0953-8984/25/i=14/a=145401.

Galanzha, E. I., E. V. Shashkov, T. Kelly, J.-W. Kim et al. (2009). In vivo magnetic enrichment and multiplex photoacoustic detection of circulating tumour cells. Nat. Nanotechnol. 4, 855. URL http: //dx.doi.org/10.1038/nnano.2009.333. 
Hembury, M., C. Chiappini, S. Bertazzo, T. L. Kalber et al. (2015). Proc. Natl. Acad. Sci. U.S.A. 112(7), 1959. URL http://www.pnas.org/content/112/7/1959.abstract.

Huang, K., H. Ma, J. Liu, S. Huo et al. (2012). Size-dependent localization and penetration of ultrasmall gold nanoparticles in cancer cells, multicellular spheroids, and tumors in vivo. ACS Nano 6(5), 4483. URL http://dx.doi.org/10.1021/nn301282m.

Jensen, S. A., E. S. Day, C. H. Ko, L. A. Hurley et al. (2013). Spherical nucleic acid nanoparticle conjugates as an RNAi-based therapy for glioblastoma. Sci. Transl. Med. 5(209), 209ra152. URL http://dx.doi. org/10.1126/scitranslmed.3006839.

Kouri, F. M., L. A. Hurley, W. L. Daniel, E. S. Day et al. (2015). miR-182 integrates apoptosis, growth, and differentiation programs in glioblastoma. Genes $\mathcal{E}$ Development 29(7), 732. URL http://dx.doi. org/10.1101/gad.257394.114.

Kruse, D. E., D. N. Stephens, H. A. Lindfors, E. S. Ingham et al. (2011). A radio-frequency coupling network for heating of citrate-coated gold nanoparticles for cancer therapy: Design and analysis. IEEE Trans. Biomed. Eng. 58(7), 2002. URL http://dx.doi.org/10.1109/TBME.2011.2124460.

Kubo, R. (1962). Electronic properties of metallic fine particles. i. J. Phys. Soc. Jpn. 17(6), 975. URL http://dx.doi.org/10.1143/JPSJ.17.975.

Kubo, R. (1977). Discreteness of energy levels in small metallic particles. J. Phys. Colloques 38(C2), C2. URL https: //hal.archives-ouvertes.fr/jpa-00217053.

Leifert, A., Y. Pan-Bartnek, U. Simon and W. Jahnen-Dechent (2013). Molecularly stabilised ultrasmall gold nanoparticles: synthesis, characterization and bioactivity. Nanoscale 5, 6224. URL http://dx. doi.org/10.1039/C3NR00916E.

Letfullin, R. R., A. R. Letfullin and T. F. George (2015). Absorption efficiency and heating kinetics of nanoparticles in the RF range for selective nanotherapy of cancer. Nanomed. Nanotechnol. Biol. Med. 11(2), 413. URL http://dx.doi.org/10.1016/j.nano.2014.09.013.

Li, D., Y. S. Jung, S. Tan, H. K. Kim et al. (2011). Negligible absorption of radiofrequency radiation by colloidal gold nanoparticles. J. Colloid Interface Sci. 358(1), 47 . URL http://www. sciencedirect. com/science/article/pii/s0021979711000750.

Liu, X., H.-j. Chen, X. Chen, C. Parini et al. (2012). Low frequency heating of gold nanoparticle dispersions for non-invasive thermal therapies. Nanoscale 4, 3945. URL http://dx.doi.org/10.1039/ C2NR30166K.

Moldosanov, K. A. and A. V. Postnikov (2013). Nanoparticle for the radiofrequency hyperthermia. Patent of the Kyrgyz Republic No. 1656 dated 20 June 2013. Bulletin "Intellectualdyk Menchik - Intellectual Property" No. 8 (185), p. 27, August 29, 2014. Bishkek, 2014. (in Kyrgyz and Russian languages). URL http://patent.kg/doc/im/2014/8.pdf.

Moran, C. H., S. M. Wainerdi, T. K. Cherukuri, C. Kittrell et al. (2009). Size-dependent joule heating of gold nanoparticles using capacitively coupled radiofrequency fields. Nano Res. 2(5), 400. URL http: //dx.doi.org/10.1007/s12274-009-9048-1.

Muñoz, J. A., M. S. Lucas, L. Mauger, I. Halevy et al. (2013). Electronic structure and vibrational entropies of fcc Au-Fe alloys. Phys. Rev. B: Condens. Matter 87, 014301. URL http://link.aps.org/doi/ 10.1103/PhysRevB.87.014301.

Murdock, R. C. and S. M. Hussain (2010). Radio frequency controlled stimulation of intracellular gold or silver nanoparticle conjugates for use as potential sensors or modulators of biological function. Technical report, Defense Technical Information Center. URL http://www.dtic.mil/dtic/tr/fulltext/ u2/a535681.pdf. Interim ReportAFRL-RH-WP-TR-2010-0141 of Air Force Research Laboratory.

Nedosekin, D. A., M. A. Juratli, M. Sarimollaoglu, C. L. Moore et al. (2013). Photoacoustic and photothermal detection of circulating tumor cells, bacteria and nanoparticles in cerebrospinal fluid in vivo and ex vivo. J. Biophotonics 6(6-7), 523. URL http://dx.doi.org/10.1002/jbio.201200242.

Pan, Y., M. Bartneck and W. Jahnen-Dechent (2012). Chapter eleven - cytotoxicity of gold nanoparticles. In: N. Düsgüneş (editor), Nanomedicine Infectious Diseases, Immunotherapy, Diagnostics, Antifibrotics, Toxicology and Gene Medicine, volume 509 of Methods in Enzymology, 225 - 242. Academic Press. URL http://www.sciencedirect.com/science/article/pii/B9780123918581000125.

Pan, Y., S. Neuss, A. Leifert, M. Fischler et al. (2007). Size-dependent cytotoxicity of gold nanoparticles. Small 3(11), 1941. URL http://dx.doi.org/10.1002/smll.200700378. 
Postnikov, A. V. and K. A. Moldosanov (2012). Non-plasmonic hyperthermia: prerequisites for realization and materials. J. Nanophotonics 6, 061709. URL http://1ink.aps.org/doi/10.1117/1.JNP.6. 061709 .

Radovic-Moreno, A. F., N. Chernyak, C. C. Mader, S. Nallagatla et al. (2015). Immunomodulatory spherical nucleic acids. Proc. Natl. Acad. Sci. U.S.A. 112(13), 3892. URL http://www.pnas.org/content/ 112/13/3892.abstract.

Randeria, P. S., M. A. Seeger, X.-Q. Wang, H. Wilson et al. (2015). siRNA-based spherical nucleic acids reverse impaired wound healing in diabetic mice by ganglioside GM3 synthase knockdown. Proc. Natl. Acad. Sci. U.S.A. 112(18), 5573. URL http: / / www.pnas.org/content/112/18/5573.abstract.

Raoof, M., S. J. Corr, W. D. Kaluarachchi, K. L. Massey et al. (2012). Stability of antibody-conjugated gold nanoparticles in the endolysosomal nanoenvironment: Implications for non-invasive radiofrequency-based cancer therapy. Nanomed. Nanotechnol. Biol. Med. 8(7), 1096. URL http://dx.doi.org/10.1016/ j.nano.2012.02.001.

Raoof, M. and S. A. Curley (2011). Non-invasive radiofrequency-induced targeted hyperthermia for the treatment of hepatocellular carcinoma. International Journal of Hepatology 2011, 676957. URL http: //dx.doi.org/10.4061/2011/676957.

San, B. H., S. H. Moh and K. K. Kim (2013). Investigation of the heating properties of platinum nanoparticles under a radiofrequency current. International Journal of Hyperthermia 29(2), 99. URL http://dx. doi.org/10.3109/02656736.2012.760137.

Singh, R. N. and I. Ali (2013). Elastic moduli and phonon dispersion curves for amorphous metals and alloys. International Journal of Applied Physics and Mathematics 3(4), 275. URL http://dx.doi. org/10.7763/IJAPM.2013.V3.220

Sirotkina, M. A., V. V. Elagin, A. A. Makarova, L. B. Snopova et al. (2012). The study of combined effect of microwave energy and gold nanoparticles on tumour in experiment. Sovrem. Technol. Med. (Modern Technologies in Medicine) 4, 30 . URL http://cyberleninka.ru/article/n/ izuchenie-sochetannogo-vozdeystviya-energii-svch-i-zolotyh-nanochastits-naopuholi-v-eksperimente.pdf. (in Russian).

Stewart, G. R. (1984). Heavy-fermion systems. Rev. Mod. Phys. 56, 755. URL http://link.aps.org/ doi/10.1103/RevModPhys.56.755

Sun, D. Y., X. G. Gong and X.-Q. Wang (2001). Soft and hard shells in metallic nanocrystals. Phys. Rev. B: Condens. Matter 63, 193412. URL http://link.aps.org/doi/10.1103/PhysRevB.63.193412.

Sun, J., L. He, Y.-C. Lo, T. Xu et al. (2014). Liquid-like pseudoelasticity of sub-10-nm crystalline silver particles. Nat. Mater. 13, 1007. URL http://dx.doi.org/10.1038/nmat 4105

Tsai, P.-C., O. Y. Chen, Y.-K. Tzeng, Y. Y. Hui et al. (2015). Gold/diamond nanohybrids for quantum sensing applications. EPJ Quantum Technology 2, 19. URL http://www . ep jquantumtechnology • com/content/2/1/19.

Wert, C. A. and R. M. Thomson (1970). Physics of Solids. McGraw-Hill series in materials science and engineering. McGraw-Hill, 2d edition edition. 